Research Article

\title{
Estimation of Integral Inequalities Using the Generalized Fractional Derivative Operator in the Hilfer Sense
}

\author{
Saima Rashid, ${ }^{1}$ Rehana Ashraf, ${ }^{2}$ Kottakkaran Sooppy Nisar, ${ }^{3}$ and Thabet Abdeljawad $\mathbb{D}^{4,5,6}$ \\ ${ }^{1}$ Department of Mathematics, Government College University, Faisalabad, Pakistan \\ ${ }^{2}$ Department of Mathematics, University of Jhang, Jhang, Pakistan \\ ${ }^{3}$ Department of Mathematics, College of Arts and Sciences, Prince Sattam bin Abdulaziz University, \\ Wadi Aldawser 11991, Saudi Arabia \\ ${ }^{4}$ Department of Mathematics and General Sciences, Prince Sultan University, Riyadh, Saudi Arabia \\ ${ }^{5}$ Department of Medical Research, China Medical University, Taiwan 40402 Taichung, Taiwan \\ ${ }^{6}$ Department of Computer Science and Information Engineering, Asia University, Taichung, Taiwan
}

Correspondence should be addressed to Thabet Abdeljawad; tabdeljawad@psu.edu.sa

Received 23 August 2020; Revised 12 September 2020; Accepted 23 September 2020; Published 27 October 2020

Academic Editor: Imtiaz Ahmad

Copyright (c) 2020 Saima Rashid et al. This is an open access article distributed under the Creative Commons Attribution License, which permits unrestricted use, distribution, and reproduction in any medium, provided the original work is properly cited.

With the great progress of fractional calculus, integral inequalities have been greatly enriched by fractional operators; users and researchers have formed a real-world phenomenon in the production of the evaluation process, which results in convexity. Monotonicity and inequality theory has a strong relationship, whichever we work on, and we can apply it to the other one due to the strong correlation produced between them, especially in the past few years. In this article, we introduce some estimations of left and right sides of the generalized Caputo fractional derivatives of a function for $n$th order differentiability via convex function, and related inequalities have been presented. Monotonicity and convexity of functions are used with some usual and straightforward inequalities. Moreover, we establish some new inequalities for $C$ eby $\breve{s}$ ev and Grüss type involving the generalized Caputo fractional derivative operators. The finding provides the theoretical basis and practical significance for the establishment of fractional calculus in convexity. It also introduces new ways of thinking and methods for innovative scientific research.

\section{Introduction}

The field of fractional calculus deals with the integrals and differentiation of arbitrary noninteger order. In the last three centuries, this field has been considered as the most powerful tool in describing anomalous kinetics and its wide applications in diverse domains. Numerous phenomena such as mathematics, statistics, engineering, physics, chemistry, and biology can be modeled by utilizing ordinary differential equations involving fractional derivatives. Many mathematicians and physicists have contributed to the development of the theories of fractional calculus [1-11]. In practical applications, various types of fractional integrals and derivative operators such as Riemann-Liouville, Caputo, Riesz, Hilfer, Hadamard, Erdelyi-Kober, Saigo, and Marichev-Saigo-Maeda were extensively studied by various researchers, see [12-17].
Later on, the mathematicians introduced the notion of fractional conformable integrals and derivatives which are cited therein. Khalil et al. [18] introduced fractional conformable derivatives operators with some shortcomings. Abdeljawad [19] investigated the properties of the fractional conformable derivative operators. Jarad et al. [20] defined generalized fractional conformable integral and derivative operators. In [21], Abdeljawad and Baleanu gave certain monotonicity results for fractional difference operators with discrete exponential kernels. Almeida [22] proposed Caputo fractional derivative in the sense of another function $\vartheta$, and in [1], the authors contemplated the idea of Riemann-Liouville fractional integrals in the sense of another function $\vartheta$. In [23], Atangana and Baleanu defined new fractional derivative operator with the nonlocal and nonsingular kernel. 
Inequalities concerning functions of two or several independent variables play an essential role in the continuous development of the theory and applications of differential and integral equations. Currently, distinctive versions of such inequalities had been developed which can be useful in the study of various classes of differential and integral equations. Those inequalities act as a far-reaching tool to study plasma physics, robotics, automatic control and many other branches of pure and applied sciences, and differential and integral equations $[24,25]$.

Convex functions are very useful in the mathematical analysis due to their fascinating properties and convenient characterizations.

Definition 1. A function $\mathscr{U}: \mathscr{I} \longrightarrow \mathbb{R}$ is said to be convex function, if the following inequality holds:

$$
\mathcal{U}\left(\zeta \tau_{1}+(1-\zeta) \tau_{2}\right) \leq \zeta \mathscr{U}\left(\tau_{1}\right)+(1-\zeta) \mathcal{U}\left(\tau_{2}\right),
$$

for all $\tau_{1}, \tau_{2} \in \mathscr{I}$ and $\zeta \in[0,1]$. If inequality (1) holds in the reverse order, then the function $\mathscr{U}$ is called concave function.

For convex functions, many equalities or inequalities have been established by many authors; for example, Hardytype inequality, Ostrowski-type inequality, and Gagliardo-Nirenberg-type inequality, but the most celebrated and significant inequality is the Hermite-Hadamard-type inequality [26-29], which is defined as

$$
\mathscr{U}\left(\frac{\tau_{1}+\tau_{2}}{2}\right) \leq \frac{1}{\tau_{2}-\tau_{1}} \int_{\tau_{1}}^{\tau_{2}} \mathscr{U}(x) \mathrm{d} x \leq \frac{\mathscr{U}\left(\tau_{1}\right)+\mathscr{U}\left(\tau_{2}\right)}{2} .
$$

A number of mathematicians in the field of applied and pure mathematics have dedicated their efforts to extend, generalize, counterpart, and refine the Hermite-Hadamard inequality (2) for different classes of convex functions. For more recent results obtained on inequality (2), we refer the reader to references [30-35].

Inspired by the aforementioned development, we propose a famous approach of generalized fractional derivative investigated in $[1,22]$, especially Caputo fractional derivative in the 9 -Hilfer sense is being utilized widely and furthermore, effectively utilized in numerous parts of sciences and engineering, see [36, 37]. Our concern is to utilize the convexity property of functions and use the absolute of their derivatives in obtaining the bounds for generalized Caputo fractional derivative presented by Definition 2.3. The new derivative is used to model the world, and we are capable of seeing that the choice of the generalized Caputo fractional derivative operator is essential for the efficiency of the numerical methods, fractional differential equations, and fractional integrodifferential equations.

It is widely recognized that $\breve{C}$ ebysev and Grüss type inequalities in continuous and discrete cases which play a significant role in studying the qualitative conduct of differential and difference equations, respectively, in addition to many other areas of mathematics. Inspired by $\breve{C}$ eby $\breve{s}$ ev [38] and Grüss [39], our aim is to show more general versions of $\breve{C}$ ebys ev and Grüss type inequalities.
$\breve{C}$ ebys ev [38] introduced the well-known celebrated functional and is defined as follows:

$$
\begin{aligned}
& \mathfrak{I}(\mathscr{U}, \mathscr{V})=\frac{1}{\tau_{2}-\tau_{1}} \int_{\tau_{1}}^{\tau_{2}} \mathscr{U}(\lambda) \mathscr{V}(\lambda) \mathrm{d} \lambda \\
& \quad-\left(\frac{1}{\tau_{2}-\tau_{1}} \int_{\tau_{1}}^{\tau_{2}} \mathscr{U}(\lambda) \mathrm{d} \lambda\right)\left(\frac{1}{\tau_{2}-\tau_{1}} \int_{\tau_{1}}^{\tau_{2}} \mathscr{V}(\lambda) \mathrm{d} \lambda\right),
\end{aligned}
$$

where $\mathcal{U}$ and $\mathscr{V}$ are two integrable functions on $\left[\tau_{1}, \tau_{2}\right]$. If $\mathscr{U}$ and $\mathscr{V}$ are synchronous, i.e.,

$$
(\mathcal{U}(\lambda)-\mathscr{U}(\omega))(\mathscr{V}(\lambda)-\mathscr{V}(\omega)) \geq 0,
$$

for any $\lambda, \omega \in\left[\tau_{1}, \tau_{2}\right]$, then $s(\mathscr{U}, \mathscr{V}) \geq 0$.

Functional (3) has vast applications in probability, numerical analysis, quantum, and statistical theory. Alongside facet with numerous applications, the functional (3) has gained plenty of interest to yield a variety of fundamental inequalities (see, for example, [40-42]).

Another interesting and fascinating aspect of the theory of inequalities is the Grüss type inequality [39] stated as follows:

$$
|s(\mathcal{U}, \mathscr{V})| \leq \frac{(Q-q)(R-r)}{4}
$$

where two integrable functions $\mathscr{U}$ and $\mathscr{V}$ on $\left[\tau_{1}, \tau_{2}\right]$, and fulfill the following:

$$
\begin{aligned}
& q \leq \mathscr{U}(\lambda) \leq Q, \\
& r \leq \mathscr{V}(\lambda) \leq R,
\end{aligned}
$$

for all $\lambda \in\left[\tau_{1}, \tau_{2}\right]$ and for some $q, Q, r, R \in \mathbb{R}$.

Many famous versions mentioned in the literature are direct effects of the numerous applications in optimizations and transform theory, see [24, 25, 42-51].

The principal aim of the present paper is to establish new bounds of some of the left-sided and right-sided Caputo fractional derivatives in Hilfer sense via convex functions that have been established. Some related inequalities via convexity and monotonicity of used functions have been proved. Moreover, the novel version of Grüss and $\breve{C}$ ebys ev types integral inequalities associated with Caputo fractional derivative operators in Hilfer sense are established for $n$th order differentiability of functions. We provide innovative special cases using a Caputo fractional derivative operator in Hilfer sense related to (3) and (5). Consequently, the effects furnished on this research paper are more generalized and may be useful in the study of fractional integral operators.

\section{Preliminaries}

In this sequel, we introduce a few notations and definitions of fractional calculus and present initial results wished in our proofs later.

Definition 2. (see $[1,2])$. A function $\mathcal{U}(\lambda)$ is said to be in $L_{p, r}[0, \infty]$ space if 


$$
L_{p, r}[0, \infty)=\left\{\mathscr{U}:\|\mathcal{U}\|_{L_{p, r}[0, \infty)}=\left(\int_{v_{1}}^{v_{2}}|\mathcal{U}(\lambda)|^{p} \lambda^{r} \mathrm{~d} \lambda\right)^{(1 / p)}<\infty, \quad 1 \leq p<\infty, r \geq 0\right\} .
$$

For $r=0$,

$$
L_{p}[0, \infty)=\left\{\mathcal{U}:\|\mathscr{U}\|_{L_{p}[0, \infty)}=\left(\int_{v_{1}}^{v_{2}}|\mathcal{U}(\lambda)|^{p} \mathrm{~d} \lambda\right)^{(1 / p)}<\infty, \quad 1 \leq p<\infty\right\} .
$$

Definition 3. (see [52]). Let $\mathscr{U} \in L_{1}[0, \infty)$ and $\vartheta$ be an increasing and positive monotone function on $[0, \infty)$ and also derivative $\mathcal{\vartheta}^{\prime}$ is continuous on $[0, \infty)$ and $\vartheta(0)=0$. The space $\chi_{9}^{p}(0, \infty)(1 \leq p<\infty)$ of those real-valued Lebesgue measurable functions $\mathscr{U}$ on $[0, \infty)$ for which

$$
\|\mathscr{U}\|_{\chi_{9}^{p}}=\left(\int_{0}^{\infty}|\mathcal{U}(\lambda)|^{p} \vartheta^{\prime}(\lambda) \mathrm{d} \lambda\right)^{(1 / p)}<\infty, \quad 1 \leq p<\infty
$$

and for the case $p=\infty$

$$
\|\mathcal{U}\|_{\chi_{9}^{\infty}}=\operatorname{ess} \sup _{0 \leq \lambda<\infty}\left[\vartheta^{\prime}(\lambda) \mathcal{U}(\lambda)\right] .
$$

In particular, when $\vartheta(\lambda)=\lambda,(1 \leq p<\infty)$, the space $\chi_{9}^{p}(0, \infty)$ coincides with the $L_{p}[0, \infty)$-space, and furthermore, if we take $\vartheta(\lambda)=\ln \lambda,(1 \leq p<\infty)$, the space $\chi_{\vartheta}^{p}(0, \infty)$ concurs with $L_{p, u}[1, \infty)$-space.

Definition 4. (see $[1,22])$. Let $\left(\tau_{1}, \tau_{2}\right)\left(-\infty \leq \tau_{1}<\tau_{2} \leq \infty\right)$ be a finite or infinite real interval and $\varrho>0$. Let $\vartheta(\zeta)$ be an increasing and positive monotone function on $\left(\tau_{1}, \tau_{2}\right]$. Then, the left Caputo fractional derivative in the 9 -Hilfer sense of order $\varrho>0$ is given by

$$
{ }^{c} \mathscr{D}_{\tau_{1}^{+}}^{\mathrm{e}, \vartheta} \mathcal{U}(\lambda):=I_{\tau_{1}^{+}}^{n-0,9}\left(\frac{1}{\mathfrak{\vartheta}^{\prime}(\lambda)} \frac{\mathrm{d}}{\mathrm{d} \lambda}\right)^{n} \mathcal{U}(\lambda),
$$

and the right Caputo fractional derivative in the 9 -Hilfer sense of $\mathscr{U}$ by

$$
{ }^{c} \mathscr{D}_{\tau_{2}^{-}}^{\mathrm{Q}, 9} \mathcal{U}(\lambda):=I_{\tau_{2}^{-}}^{n-0, \vartheta}\left(-\frac{1}{\vartheta^{\prime}(\lambda)} \frac{\mathrm{d}}{\mathrm{d} \lambda}\right)^{n} \mathcal{U}(\lambda),
$$

where $n=[\varrho]+1$ for $\varrho \notin \mathbb{N}, \quad n=\varrho$ for $\varrho \in \mathbb{N}$ and if $\varrho \notin \mathbb{N}$; then,

$$
\begin{gathered}
{ }^{c} \mathscr{D}_{\tau_{1}^{+}}^{\rho, 9} \mathscr{U}(\lambda)=\frac{1}{\Gamma(n-\varrho)} \int_{\tau_{1}}^{\lambda} \vartheta^{\prime}(\zeta)(\vartheta(\lambda)-\vartheta(\zeta))^{n-\varrho-1} \mathcal{U}_{\vartheta}^{[n]}(\zeta) \mathrm{d} \zeta, \\
{ }^{c} \mathscr{D}_{\tau_{2}^{0}}^{\rho, 9} \mathcal{U}(\lambda)=\frac{(-1)^{n}}{\Gamma(n-\varrho)} \int_{\lambda}^{\tau_{2}} \vartheta^{\prime}(\zeta)(\vartheta(\zeta)-\vartheta(\lambda))^{n-\varrho-1} \mathcal{U}_{\vartheta}^{[n]}(\zeta) \mathrm{d} \zeta,
\end{gathered}
$$

where $\Gamma(z)=\int_{0}^{\infty} \zeta^{z-1} e^{-\zeta} \mathrm{d} \zeta$ is the Euler gamma function.

Remark 1. It can be easily noticed that

(1) When $\vartheta(\zeta)=\zeta$, then (13) and (14) are the classical Caputo derivative [1]

(2) When $\vartheta(\zeta)=\ln \zeta$, then (13) and (14) are the Caputo-Hadamard fractional derivative [43]

(3) when $\vartheta(\zeta)=\zeta^{\rho} / \rho$, then (13) and (14) are the Caputo modification of the left and right generalized fractional derivatives in the sense of [53]

(4) When $\vartheta(\zeta)=(\zeta-a)^{\rho} / \rho$, then (13) and (14) are the fractional conformable derivative in the sense of [20]

Now, we present a one-sided fractional operator which is known as the generalized Caputo fractional derivative operator.

Definition 5. Let $\left(\tau_{1}, \tau_{2}\right)\left(-\infty \leq \tau_{1}<\tau_{2} \leq \infty\right)$ be a finite or infinite real interval and $\varrho>0$. Let $\vartheta(\zeta)$ be an increasing and positive monotone function on $\left(\operatorname{tau}_{1}, \operatorname{tau}_{2}\right]$. Then, the leftsided and right-sided Caputo fractional derivative in the $\vartheta$-Hilfer sense of order $\varrho$ is defined as follows:

$$
\mathscr{D}_{0^{+}}^{9, \mathrm{e}} \mathscr{U}(\lambda)=\frac{1}{\Gamma(n-\varrho)} \int_{0}^{\lambda} \vartheta^{\prime}(\zeta)(\vartheta(\lambda)-\vartheta(\zeta))^{n-\varrho^{-1}} \mathcal{U}(\zeta) \mathrm{d} \zeta .
$$

\section{Hermite-Hadamard-Type Inequalities for Caputo Fractional Derivative in the 9-Hilfer Sense}

Theorem 1. For $n \in \mathbb{N}, \varrho, \delta \geq 1$, and let there be a realvalued $n$-times differentiable function $\mathscr{U}: \mathscr{I}=\left[\tau_{1}\right.$, $\left.\tau_{2}\right] \longrightarrow \mathbb{R}$ defined on $\mathcal{I}$. Also, assume that $\vartheta$ be differentiable and strictly increasing such that with $\vartheta^{\prime} \in L_{1}\left(\left[\tau_{1}, \tau_{2}\right]\right)$. If $\vartheta^{(n)}$ is a convex function on $\mathscr{F}$, for all $\tau_{1}, \tau_{2} \in \mathscr{I}$ and $\tau_{1}<\tau_{2}$, then 


$$
\begin{aligned}
\Gamma & (n-\varrho+1)\left({ }^{c} \mathscr{D}_{\tau_{1}^{+}}^{\varrho-1, \vartheta} \mathcal{U}\right)(\lambda)+\Gamma(n-\delta+1)\left({ }^{c} \mathscr{D}_{\tau_{2}^{-}}^{\varrho-1, \vartheta} \mathcal{U}\right)(\lambda) \\
\leq & \frac{\left(\vartheta(\lambda)-\vartheta\left(\tau_{1}\right)\right)^{n-\varrho}}{\lambda-\tau_{1}}\left[\left(\lambda-\tau_{1}\right)\right. \\
& {\left[\mathcal{U}^{(n)}(\lambda) \vartheta(\lambda)-\mathcal{U}^{(n)}\left(\tau_{1}\right) \vartheta\left(\tau_{1}\right)\right] } \\
& \left.-\left(\mathcal{U}^{(n)}(\lambda)-\mathcal{U}^{(n)}\left(\tau_{1}\right)\right) \int_{\tau_{1}}^{\lambda} \vartheta(\zeta) \mathrm{d} \zeta\right] \\
& +\frac{\left(\vartheta\left(\tau_{2}\right)-\vartheta(\lambda)\right)^{n-\delta}}{\tau_{2}-\lambda}\left[\left(\tau_{2}-\lambda\right)\right. \\
& {\left[\mathcal{U}^{(n)}\left(\tau_{2}\right) \vartheta\left(\tau_{2}\right)-\mathcal{U}^{(n)}(\lambda) \vartheta(\lambda)\right] } \\
& \left.-\left(\mathcal{U}^{(n)}\left(\tau_{2}\right)-\mathcal{U}^{(n)}(\lambda)\right) \int_{\lambda}^{\tau_{2}} \vartheta(\zeta) \mathrm{d} \zeta\right] .
\end{aligned}
$$

Proof. Utilizing the given hypothesis, we have

$$
(\vartheta(\lambda)-\vartheta(\zeta))^{n-\varrho} \leq\left(\vartheta(\lambda)-\vartheta\left(\tau_{1}\right)\right)^{n-\varrho},
$$

where $\lambda \in\left[\tau_{1}, \tau_{2}\right]$ and $\zeta \in\left[\tau_{1}, \tau_{2}\right], \varrho \geq 1$, and $\vartheta^{\prime}(\zeta)>0$. Hence, the following inequality holds true:

$\vartheta^{\prime}(\zeta)(\vartheta(\lambda)-\vartheta(\zeta))^{n-\varrho} \leq \vartheta^{\prime}(\zeta)\left(\vartheta(\lambda)-\vartheta\left(\tau_{1}\right)\right)^{n-\varrho}$.

By the convexity of $\mathscr{U}^{(n)}$, we have

$$
\mathcal{U}^{(n)}(\zeta) \leq \frac{\lambda-\zeta}{\lambda-\tau_{1}} \mathcal{U}^{(n)}\left(\tau_{1}\right)+\frac{\zeta-\tau_{1}}{\lambda-\tau_{1}} \mathcal{U}^{(n)}(\lambda) .
$$

From (18) and (19), one has

$$
\begin{aligned}
& \int_{\tau_{1}}^{\lambda}(\vartheta(\lambda)-\vartheta(\zeta))^{n-e} \mathcal{U}^{(n)}(\zeta) \vartheta^{\prime}(\zeta) \mathrm{d} \zeta \\
& \leq \frac{\left(\vartheta(\lambda)-\vartheta\left(\tau_{1}\right)\right)^{n-\varrho}}{\lambda-\tau_{1}}\left[\mathcal{U}^{(n)}\left(\tau_{1}\right) \int_{\tau_{1}}^{\lambda}(\lambda-\zeta)+\mathcal{U}^{(n)}(\lambda)\right. \\
& \left.\quad \int_{\tau_{1}}^{\lambda}\left(\zeta-\tau_{1}\right) \vartheta^{\prime}(\zeta) \mathrm{d} \zeta\right] .
\end{aligned}
$$

Using (13) from Definition 4, we obtain

$$
\begin{aligned}
& \Gamma(n-\varrho+1)\left({ }^{c} \mathscr{D}_{\tau_{1}^{+}}^{\varrho-1, \vartheta} \mathcal{U}\right)(\lambda) \\
& \leq \frac{\left(\vartheta(\lambda)-\vartheta\left(\tau_{1}\right)\right)^{n-\varrho}}{\lambda-\tau_{1}}\left[( \lambda - \tau _ { 1 } ) \left[\mathcal{U}^{(n)}(\lambda) \vartheta(\lambda)\right.\right. \\
& \left.\left.\quad-\mathcal{U}^{(n)}\left(\tau_{1}\right) \vartheta\left(\tau_{1}\right)\right]-\left(\mathcal{U}^{(n)}(\lambda)-\mathcal{U}^{(n)}\left(\tau_{1}\right)\right) \int_{\tau_{1}}^{\lambda} \vartheta(\zeta) \mathrm{d} \zeta\right] .
\end{aligned}
$$

Now, for $\lambda \in\left[\tau_{1}, \tau_{2}\right], \zeta \in\left[\lambda, \tau_{2}\right]$, and $\delta \geq 1$, the following inequality holds true:

$$
\vartheta^{\prime}(\zeta)(\vartheta(\zeta)-\vartheta(\lambda))^{n-\delta} \leq \vartheta^{\prime}(\zeta)\left(\vartheta\left(\tau_{2}\right)-\vartheta(\lambda)\right)^{n-\delta} .
$$

Utilizing convexity of $\mathscr{U}^{(n)}$, it follows that

$$
\mathcal{U}^{(n)}(\zeta) \leq \frac{\zeta-\lambda}{\tau_{2}-\lambda} \mathcal{U}^{(n)}\left(\tau_{2}\right)+\frac{\tau_{2}-\zeta}{\tau_{2}-\lambda} \mathcal{U}^{(n)}(\lambda)
$$

Repeating the same procedure as we have done for (18) and (19), one can acquire from (22) and (23); then,

$$
\begin{gathered}
\Gamma(n-\delta+1)\left({ }^{c} \mathscr{D}_{\tau_{2}^{-}}^{\delta-1,9} \mathcal{U}\right)(\lambda) \leq \frac{\left(\vartheta\left(\tau_{2}\right)-\vartheta(\lambda)\right)^{n-\delta}}{\tau_{2}-\lambda} \\
\quad \times\left[\left(\tau_{2}-\lambda\right)\left[\mathcal{U}^{(n)}\left(\tau_{2}\right) \vartheta\left(\tau_{2}\right)-\mathscr{U}^{(n)}(\lambda) \vartheta(\lambda)\right]\right. \\
\left.-\left(\mathcal{U}^{(n)}\left(\tau_{2}\right)-\mathcal{U}^{(n)}(\lambda)\right) \int_{\lambda}^{\tau_{2}} \vartheta(\zeta) \mathrm{d} \zeta\right] .
\end{gathered}
$$

From inequalities (21) and (24), we get (16) which is required.

Corollary 1. If we take $\alpha=\delta$ in (16), we get the result for generalized Caputo fractional derivative operator:

$$
\begin{aligned}
& \Gamma(n-\alpha+1)\left[\left({ }^{c} \mathscr{D}_{\tau_{1}^{+}}^{\alpha-1, \vartheta} \mathcal{U}\right)(\lambda)+\left({ }^{c} \mathscr{D}_{\tau_{2}^{-}}^{\alpha-9} \mathcal{U}\right)(\lambda)\right] \\
& \leq \frac{\left(\vartheta(\lambda)-\vartheta\left(\tau_{1}\right)\right)^{n-\alpha}}{\lambda-\tau_{1}}\left[( \lambda - \tau _ { 1 } ) \left[\mathcal{U}^{(n)}(\lambda) \vartheta(\lambda)\right.\right. \\
& \left.\left.\quad-\mathcal{U}^{(n)}\left(\tau_{1}\right) \vartheta\left(\tau_{1}\right)\right]-\left(\mathcal{U}^{(n)}(\lambda)-\mathcal{U}^{(n)}\left(\tau_{1}\right)\right) \int_{\tau_{1}}^{\lambda} \vartheta(\zeta) \mathrm{d} \zeta\right] \\
& +\frac{\left(\vartheta\left(\tau_{2}\right)-\vartheta(\lambda)\right)^{n-\alpha}}{\tau_{2}-\lambda}\left[( \tau _ { 2 } - \lambda ) \left[\mathcal{U}^{(n)}\left(\tau_{2}\right) \vartheta\left(\tau_{2}\right)\right.\right. \\
& \left.\left.-\mathscr{U}^{(n)}(\lambda) \vartheta(\lambda)\right]-\left(\mathcal{U}^{(n)}\left(\tau_{2}\right)-\mathscr{U}^{(n)}(\lambda)\right) \int_{\lambda}^{\tau_{2}} \vartheta(\zeta) \mathrm{d} \zeta\right] .
\end{aligned}
$$

Theorem 2. For $n \in \mathbb{N}, \varrho, \delta \geq 1$, and let there be a real-valued $n$-times differentiable function $\mathcal{U}: \mathscr{I}=\left[\tau_{1}, \tau_{2}\right] \longrightarrow \mathbb{R}$ defined on $\mathscr{U}: \mathscr{I}=\left[\tau_{1}, \tau_{2}\right] \longrightarrow \mathbb{R}$. Also, assume that $\vartheta$ be differentiable and strictly increasing such that with $\vartheta^{\prime} \in L_{1}\left(\left[\tau_{1}, \tau_{2}\right]\right)$. If $\left|\vartheta^{(n+1)}\right|$ is a convex function on $\mathcal{F}$, for all $\tau_{1}, \tau_{2} \in \mathscr{I}$ and $\tau_{1}<\tau_{2}$, then 


$$
\begin{aligned}
& \mid \begin{array}{l}
\Gamma(n-\varrho+1)\left({ }^{c} \mathscr{D}_{\tau_{1}^{+}}^{\mathrm{e}, \vartheta} \mathcal{U}\right)(\lambda)+\Gamma(n-\delta+1)\left({ }^{c} \mathscr{D}_{\tau_{2}^{-}}^{\mathrm{e}, \vartheta} \mathcal{U}\right)(\lambda) \\
-\left[\left(\vartheta(\lambda)-\vartheta\left(\tau_{1}\right)\right)^{n-\varrho} \mathcal{U}^{(n)}\left(\tau_{1}\right)+\left(\vartheta\left(\tau_{2}\right)-\vartheta(\lambda)\right)^{n-\delta} \mathcal{U}^{(n)}\left(\tau_{2}\right)\right] \mid
\end{array} \\
& \leq \frac{\left(\vartheta(\lambda)-\vartheta\left(\tau_{1}\right)\right)^{n-\varrho}\left(\lambda-\tau_{1}\right)\left|\mathcal{U}^{(n+1)}\left(\tau_{1}\right)+\left(\vartheta\left(\tau_{2}\right)-\vartheta(\lambda)\right)^{n-\delta}\left(\tau_{2}-\lambda\right)\right| \mathcal{U}^{(n+1)}\left(\tau_{2}\right) \mid}{2} \\
& +\left|\mathcal{U}^{(n+1)}(\lambda)\right| \frac{\left(\vartheta(\lambda)-\vartheta\left(\tau_{1}\right)\right)^{n-\varrho}\left(\lambda-\tau_{1}\right)+\left(\vartheta\left(\tau_{2}\right)-\vartheta(\lambda)\right)^{n-\delta}\left(\tau_{2}-\lambda\right)}{2} .
\end{aligned}
$$

Proof. From convexity of $\left|\mathcal{U}^{(n+1)}\right|$, we have

$$
\left|\mathcal{U}^{(n+1)}(\zeta)\right| \leq \frac{\lambda-\zeta}{\lambda-\tau_{1}}\left|\mathcal{U}^{(n+1)}\left(\tau_{1}\right)\right|+\frac{\zeta-\tau_{1}}{\lambda-\tau_{1}}\left|\mathcal{U}^{(n+1)}(\lambda)\right| .
$$

From (27), one obtains

$$
\mathscr{U}^{(n+1)}(\zeta) \leq \frac{\lambda-\zeta}{\lambda-\tau_{1}}\left|\mathscr{U}^{(n+1)}\left(\tau_{1}\right)\right|+\frac{\zeta-\tau_{1}}{\lambda-\tau_{1}}\left|\mathcal{U}^{(n+1)}(\lambda)\right| .
$$

Since the function 9 is differentiable and strictly increasing, therefore we have the following inequality:

$$
(\vartheta(\lambda)-\vartheta(\zeta))^{n-\varrho} \leq\left(\vartheta(\lambda)-\vartheta\left(\tau_{1}\right)\right)^{n-\varrho},
$$

where $\lambda \in\left[\tau_{1}, \tau_{2}\right], \zeta \in\left[\tau_{1}, \lambda\right]$, and $\varrho>0$.

From (28) and (29), one has

$$
\begin{aligned}
& (\vartheta(\lambda)-\vartheta(\zeta))^{n-\varrho} \mathcal{U}^{(n+1)}(\zeta) \\
& \leq \frac{\left(\vartheta(\lambda)-\vartheta\left(\tau_{1}\right)\right)^{n-\varrho}}{\lambda-\tau_{1}}\left\{(\lambda-\zeta)\left|\mathcal{U}^{(n+1)}\left(\tau_{1}\right)\right|\right. \\
& \left.\quad+\left(\zeta-\tau_{1}\right)\left|\mathcal{U}^{(n+1)}(\lambda)\right|\right\} .
\end{aligned}
$$

Integrating over $\left[\tau_{1}, \lambda\right]$, we have

$$
\begin{aligned}
& \int_{\tau_{1}}^{\lambda}(\vartheta(\lambda)-\vartheta(\zeta))^{n-\varrho} \mathcal{U}^{(n+1)}(\zeta) \mathrm{d} \zeta \\
& \leq \frac{\left(\vartheta(\lambda)-\vartheta\left(\tau_{1}\right)\right)^{n-\varrho}}{\lambda-\tau_{1}}\left\{\left|\mathcal{U}^{(n+1)}\left(\tau_{1}\right)\right| \int_{\tau_{1}}^{\lambda}(\lambda-\zeta) \mathrm{d} \zeta\right. \\
& \left.+\left|\mathcal{U}^{(n+1)}(\lambda)\right| \int_{\tau_{1}}^{\lambda}\left(\zeta-\tau_{1}\right) \mathrm{d} \zeta\right\} \\
& \left.=\left(\vartheta(\lambda)-\vartheta\left(\tau_{1}\right)\right)^{n-\varrho}\left(\lambda-\tau_{1}\right) \mid \frac{\left.\left|\mathcal{U}^{(n+1)}\left(\tau_{1}\right)\right|+\left|\mathcal{U}^{(n+1)}(\lambda)\right|\right]}{2}\right] \\
& \quad \int_{\tau_{1}}^{\lambda}\left(\vartheta(\lambda)-\vartheta\left(\tau_{1}\right)\right)^{n-\varrho} \mathcal{U}^{(n+1)}(\zeta) \mathrm{d} \zeta \\
& =\left.\mathcal{U}^{(n)}(\zeta)\left(\vartheta(\lambda)-\vartheta\left(\tau_{1}\right)\right)^{n-\varrho}\right|_{\tau_{1}} ^{\lambda}+(n-\varrho) \\
& \quad \int_{\tau_{1}}^{\lambda}\left(\vartheta(\lambda)-\vartheta\left(\tau_{1}\right)\right)^{n-\varrho-1} \vartheta^{\prime}(\zeta) \mathcal{U}^{(n)}(\zeta) \mathrm{d} \zeta \\
& =-\mathscr{U}^{(n)}\left(\tau_{1}\right)\left(\vartheta(\lambda)-\vartheta\left(\tau_{1}\right)\right)^{n-\varrho} \\
& \quad+\Gamma(n-\varrho+1)\left({ }^{c} \mathscr{D}_{\tau_{1}^{+}}^{\varrho, \vartheta} \mathcal{U}^{(n)}(\lambda) .\right.
\end{aligned}
$$

From (31), it follows that

$$
\begin{aligned}
& \Gamma(n-\varrho+1)\left({ }^{c} \mathscr{D}_{\tau_{1}^{+}}^{\varrho, \vartheta} \mathcal{U}\right)(\lambda)-\mathcal{U}^{(n)}\left(\tau_{1}\right)\left(\vartheta(\lambda)-\vartheta\left(\tau_{1}\right)\right)^{n-\varrho} \\
& \leq\left(\vartheta(\lambda)-\vartheta\left(\tau_{1}\right)\right)^{n-\varrho}\left(\lambda-\tau_{1}\right)\left[\frac{\left|U^{(n+1)}\left(\tau_{1}\right)\right|+\left|\mathcal{U}^{(n+1)}(\lambda)\right|}{2}\right] .
\end{aligned}
$$

Also, from (27), one has

$$
\mathcal{U}^{(n+1)}(\zeta) \geq\left(\frac{\lambda-\zeta}{\lambda-\tau_{1}}\left|\mathcal{U}^{(n+1)}\left(\tau_{1}\right)\right|+\frac{\zeta-\tau_{1}}{\lambda-\tau_{1}}\left|\mathcal{U}^{(n+1)}(\lambda)\right|\right) \text {. }
$$

Repeating the same procedure as we did for (28), we have

$$
\begin{aligned}
& \mathcal{U}^{(n)}\left(\tau_{1}\right)\left[\left(\vartheta(\lambda)-\vartheta\left(\tau_{1}\right)\right)^{n-\varrho}\right]-\Gamma(n-\varrho+1)\left({ }^{c} \mathscr{D}_{\tau_{1}^{+}}^{\varrho, \vartheta} \mathcal{U}\right)(\lambda) \\
& \leq\left(\vartheta(\lambda)-\vartheta\left(\tau_{1}\right)\right)^{n-\varrho}\left(\lambda-\tau_{1}\right)\left[\frac{\left|\mathcal{U}^{(n+1)}\left(\tau_{1}\right)\right|+\left|\mathcal{U}^{(n+1)}(\lambda)\right|}{2}\right] .
\end{aligned}
$$

From (33) and (35), we obtain

$$
\begin{aligned}
& \left|\Gamma(n-\varrho+1)\left({ }^{c} \mathscr{D}_{\tau_{1}^{+}}^{\varrho, \vartheta} f\right)(\lambda)-\mathcal{U}^{(n)}\left(\tau_{1}\right)\left[\left(\vartheta(\lambda)-\vartheta\left(\tau_{1}\right)\right)^{n-\varrho}\right]\right| \\
& \leq\left(\vartheta(\lambda)-\vartheta\left(\tau_{1}\right)\right)^{n-\varrho}\left(\lambda-\tau_{1}\right)\left[\frac{\left|\mathcal{U}^{(n+1)}\left(\tau_{1}\right)\right|+\left|\mathcal{U}^{(n+1)}(\lambda)\right|}{2}\right] .
\end{aligned}
$$

From convexity of $\left|\mathcal{U}^{(n+1)}\right|$, one obtains

$$
\left|\mathcal{U}^{(n+1)}(\zeta)\right| \leq \frac{\zeta-\lambda}{\tau_{2}-\lambda}\left|\mathcal{U}^{(n+1)}\left(\tau_{2}\right)\right|+\frac{\tau_{2}-\lambda}{\tau_{2}-\lambda}\left|\mathcal{U}^{(n+1)}(\lambda)\right|
$$

Now, for $\lambda \in\left[\tau_{1}, \tau_{2}\right]$ and $\zeta \in\left[\lambda, \tau_{2}\right]$ and $\delta>0$, the following inequality holds true:

$$
(\vartheta(\zeta)-\vartheta(\lambda))^{n-\delta} \leq\left(\vartheta\left(\tau_{2}\right)-\vartheta(\lambda)\right)^{n-\delta} .
$$

If we proceed in a similar way as we did for (28), (29), and (34), one can get from (37) and (38) the following inequality: 


$$
\begin{aligned}
& \left|\Gamma(n-\delta+1)\left({ }^{c} \mathscr{D}_{\tau_{2}^{-}}^{\delta, \vartheta} \mathcal{U}\right)(\lambda)-\mathcal{U}^{(n)}\left(\tau_{2}\right)\left[\left(\vartheta\left(\tau_{2}\right)-\vartheta(\lambda)\right)^{n-\delta}\right]\right| \\
& \leq(\vartheta(\delta)-\vartheta(\lambda))^{n-\delta}\left(\tau_{2}-\lambda\right)\left[\frac{\left|\mathcal{U}^{(n+1)}(\lambda)\right|+\left|\mathcal{U}^{(n+1)}\left(\tau_{2}\right)\right|}{2}\right] .
\end{aligned}
$$

From inequalities (36) and (39) via triangular inequality, we get (26) which is required.

Corollary 2. If we take $\mathrm{Q}=\delta$ in (26), then we get the following inequality for generalized Caputo fractional derivative operator:

$$
\begin{aligned}
& \mid \Gamma(n-\varrho+1)\left\{\left({ }^{c} \mathscr{D}_{\tau_{1}^{+}}^{\mathrm{\rho}, \vartheta} \mathcal{U}\right)(\lambda)+\left({ }^{c} \mathscr{D}_{\tau_{2}^{-}}^{\varrho, \vartheta} \mathcal{U}\right)(\lambda)\right\} \\
& \quad-\left[\left(\vartheta(\lambda)-\vartheta\left(\tau_{1}\right)\right)^{n-\varrho} \mathcal{U}^{(n)}\left(\tau_{1}\right)+\left(\vartheta\left(\tau_{2}\right)-\vartheta(\lambda)\right)^{n-\varrho} \mathcal{U}^{(n)}\left(\tau_{2}\right)\right] \mid \\
& \leq \frac{\left(\vartheta(\lambda)-\vartheta\left(\tau_{1}\right)\right)^{n-\varrho}\left(\lambda-\tau_{1}\right)\left|\mathcal{U}^{(n+1)}\left(\tau_{1}\right)+\left(\vartheta\left(\tau_{2}\right)-\vartheta(\lambda)\right)^{n-\varrho}\left(\tau_{2}-\lambda\right)\right| \mathcal{U}^{(n+1)}\left(\tau_{2}\right) \mid}{2} \\
& \quad+\left|\mathcal{U}^{(n+1)}(\lambda)\right| \frac{\left(\vartheta(\lambda)-\vartheta\left(\tau_{1}\right)\right)^{n-\varrho}\left(\lambda-\tau_{1}\right)+\left(\vartheta\left(\tau_{2}\right)-\vartheta(\lambda)\right)^{n-\varrho}\left(\tau_{2}-\lambda\right)}{2} .
\end{aligned}
$$

Lemma 1 (see [28]). Suppose that $\mathscr{U}: \mathscr{I} \longrightarrow \mathbb{R}$ is a convex function which is symmetric about $\left(\tau_{1}+\tau_{2}\right) / 2$; then, the following inequality holds:

$$
\mathscr{U}\left(\frac{\tau_{1}+\tau_{2}}{2}\right) \leq \mathscr{U}(\lambda), \quad \lambda \in\left[\tau_{1}, \tau_{2}\right] .
$$

Theorem 3. For $n \in \mathbb{N}, \varrho, \delta \geq 1$, and let there be a real-valued $n$-times differentiable function $\mathcal{U}: \mathscr{I}=\left[\tau_{1}, \tau_{2}\right] \longrightarrow \mathbb{R}$ defined on $\mathscr{F}$, where $n$ is a positive integer such that $\mathcal{U}^{(n)}$ be positive convex and symmetric about $\left(\tau_{1}+\tau_{2}\right) / 2$. Also, assume that $\vartheta$ be differentiable and strictly increasing such that with $\vartheta^{\prime} \in L_{1}\left(\left[\tau_{1}, \tau_{2}\right]\right)$ for all $\tau_{1}, \tau_{2} \in \mathscr{I}$ and $\tau_{1}<\tau_{2}$; then,

$$
\begin{aligned}
& \frac{1}{2}\left(\frac{1}{n-\varrho+1}+\frac{1}{n-\delta+1}\right) \mathcal{U}^{(n)}\left(\frac{\tau_{1}+\tau_{2}}{2}\right) \\
& \leq \frac{\Gamma(n-\varrho+1)\left({ }^{c} \mathscr{D}_{1}^{\varrho-1,9} \mathcal{U}\right)\left(\tau_{2}\right)}{2\left(\vartheta\left(\tau_{2}\right)-\vartheta\left(\tau_{1}\right)\right)^{n-\varrho+1}}+\frac{\Gamma(n-\delta+1)\left({ }^{c} \mathscr{D}_{\tau_{2}^{-}}^{\delta-1,9} \mathcal{U}\right)\left(\tau_{1}\right)}{2\left(\vartheta\left(\tau_{2}\right)-\vartheta\left(\tau_{1}\right)\right)^{n-\delta+1}} \\
& \leq \frac{1}{\left(\vartheta\left(\tau_{2}\right)-\vartheta\left(\tau_{1}\right)\right)\left(\tau_{2}-\tau_{1}\right)}\left[\left(\tau_{2}-\tau_{1}\right)\left[\mathcal{U}^{(n)}\left(\tau_{2}\right) \vartheta\left(\tau_{2}\right)-\mathcal{U}^{(n)}\left(\tau_{1}\right) \vartheta\left(\tau_{1}\right)\right]-\left(\mathcal{U}^{(n)}\left(\tau_{2}\right)-\mathcal{U}^{(n)}\left(\tau_{1}\right)\right) \int_{\tau_{1}}^{\tau_{2}} \vartheta(\lambda) \mathrm{d} \lambda\right] .
\end{aligned}
$$

Proof. Utilizing the given hypothesis, we have

$$
\left(\vartheta(\lambda)-\vartheta\left(\tau_{1}\right)\right)^{n-\delta} \leq\left(\vartheta\left(\tau_{2}\right)-\vartheta\left(\tau_{1}\right)\right)^{n-\delta},
$$

where $\lambda \in\left[\tau_{1}, \tau_{2}\right], \delta>0$, and $\vartheta^{\prime}(\lambda)>0$. Hence, the following inequality holds true:

$$
\vartheta^{\prime}(\lambda)\left(\vartheta(\lambda)-\vartheta\left(\tau_{1}\right)\right)^{n-\delta} \leq \vartheta^{\prime}\left(\vartheta\left(\tau_{2}\right)-\vartheta\left(\tau_{1}\right)\right)^{n-\delta} .
$$

By using the convexity of $\mathcal{U}^{(n)}$, we have

$$
\mathcal{U}^{(n)}(\lambda) \leq \frac{\lambda-\tau_{1}}{\tau_{2}-\tau_{1}} \mathscr{U}^{(n)}\left(\tau_{2}\right)+\frac{\tau_{2}-\lambda}{\tau_{2}-\tau_{1}} \mathcal{U}^{(n)}\left(\tau_{1}\right) .
$$

From (44) and (45), one has

$$
\begin{aligned}
& \int_{\tau_{1}}^{\tau_{2}}\left(\vartheta(\lambda)-\vartheta\left(\tau_{1}\right)\right)^{n-\delta} \mathscr{U}^{(n)}(\lambda) \vartheta^{\prime}(\lambda) \mathrm{d} \lambda \\
& \leq \frac{\left(\vartheta\left(\tau_{2}\right)-\vartheta\left(\tau_{1}\right)\right)^{n-\delta}}{\tau_{2}-\tau_{1}}\left[\mathcal{U}^{(n)}\left(\tau_{2}\right) \int_{\tau_{1}}^{\tau_{2}}\left(x-\tau_{1}\right) \vartheta^{\prime}(\lambda) \mathrm{d} \lambda\right. \\
& \left.+\mathscr{U}^{(n)}\left(\tau_{1}\right) \int_{\tau_{1}}^{\tau_{2}}\left(\tau_{2}-x\right) \vartheta^{\prime}(\lambda) \mathrm{d} \lambda\right] .
\end{aligned}
$$

Using (13) from Definition 4, we obtain 


$$
\begin{aligned}
& \Gamma(n-\delta+1)\left({ }^{c} \mathscr{D}_{\tau_{2}^{-}}^{\delta-1, \vartheta} f\right)\left(\tau_{1}\right) \\
& \leq \frac{\left(\vartheta\left(\tau_{2}\right)-\vartheta\left(\tau_{1}\right)\right)^{n-\delta}}{\tau_{2}-\tau_{1}}\left[( \tau _ { 2 } - \tau _ { 1 } ) \left[\mathscr{U}^{(n)}\left(\tau_{2}\right) \vartheta\left(\tau_{2}\right)\right.\right. \\
& \left.\left.\quad-\mathscr{U}^{(n)}\left(\tau_{1}\right) \vartheta\left(\tau_{1}\right)\right]-\left(\mathcal{U}^{(n)}\left(\tau_{2}\right)-\mathscr{U}^{(n)}\left(\tau_{1}\right)\right) \int_{\tau_{1}}^{\tau_{2}} \vartheta(\lambda) \mathrm{d} \lambda\right] .
\end{aligned}
$$

Now for $\lambda \in\left[\tau_{1}, \tau_{2}\right], \zeta \in\left[\lambda, \tau_{2}\right]$, and $\varrho>0$, the following inequality holds true:

$$
\vartheta^{\prime}(\lambda)\left(\vartheta\left(\tau_{2}\right)-\vartheta(\lambda)\right)^{n-\varrho} \leq \vartheta^{\prime}(\lambda)\left(\vartheta\left(\tau_{2}\right)-\vartheta\left(\tau_{1}\right)\right)^{n-\varrho} .
$$

By a similar argument which we have done for (44) and (45), one can get from (45) and (48) the following inequality:

$$
\begin{aligned}
& \Gamma(n-\varrho+1)\left({ }^{c} \mathscr{D}_{\tau_{1}^{+}}^{\varrho^{-1, \vartheta}} \mathcal{U}\right)\left(\tau_{2}\right) \\
& \leq \frac{\left(\vartheta\left(\tau_{2}\right)-\vartheta\left(\tau_{1}\right)\right)^{n-\varrho}}{\tau_{2}-\tau_{1}}\left[( \tau _ { 2 } - \tau _ { 1 } ) \left[\mathcal{U}^{(n)}\left(\tau_{2}\right) \vartheta\left(\tau_{2}\right)\right.\right. \\
& \left.\left.\quad-\mathscr{U}^{(n)}\left(\tau_{1}\right) \vartheta\left(\tau_{1}\right)\right]-\left(\mathcal{U}^{(n)}\left(\tau_{2}\right)-\mathcal{U}^{(n)}\left(\tau_{1}\right)\right) \int_{\tau_{1}}^{\tau_{2}} \vartheta(\lambda) \mathrm{d} \lambda\right] .
\end{aligned}
$$

Since $\mathcal{U}^{(n)}$ is convex and symmetric about $\left(\tau_{1}+\tau_{2}\right) / 2$ using Lemma 1 and multiplying (41) with $\left(\vartheta(\lambda)-\vartheta\left(\tau_{1}\right)\right)^{n-\delta} \vartheta^{\prime}(\lambda)$ and integrating over $\left[\tau_{1}, \tau_{2}\right]$, we obtain

$$
\begin{aligned}
& \mathcal{U}^{(n)}\left(\frac{\tau_{1}+\tau_{2}}{2}\right) \int_{\tau_{1}}^{\tau_{2}}\left(\vartheta(\lambda)-\vartheta\left(\tau_{1}\right)\right)^{n-\delta} \vartheta^{\prime}(\lambda) \mathrm{d} \lambda \\
& \leq \int_{\tau_{1}}^{\tau_{2}}\left(\vartheta(\lambda)-\vartheta\left(\tau_{1}\right)\right)^{n-\delta} \vartheta^{\prime}(\lambda) \mathcal{U}^{(n)}(\lambda) \mathrm{d} \lambda .
\end{aligned}
$$

Using (14) from Definition 4, we obtain

$$
\frac{\mathcal{U}^{(n)}\left(\left(\tau_{1}+\tau_{2}\right) / 2\right)}{2(n-\delta+1)} \leq \frac{\Gamma(n-\delta+1)\left({ }^{c} \mathscr{D}_{\tau_{2}^{-}}^{\delta-1, \vartheta} \mathcal{U}\right)\left(\tau_{1}\right)}{2\left(\vartheta\left(\tau_{2}\right)-\vartheta\left(\tau_{1}\right)\right)^{n-\delta+1}} .
$$

Similarly, using Lemma 1 and multiplying (41) with $\left(\vartheta\left(\tau_{2}\right)-\vartheta(\lambda)\right)^{n-\varrho} \vartheta^{\prime}(\lambda)$ and then integrating over $\left[\tau_{1}, \tau_{2}\right]$, we have

$$
\frac{\mathcal{U}^{(n)}\left(\left(\tau_{1}+\tau_{2}\right) / 2\right)}{2(n-\varrho+1)} \leq \frac{\Gamma(n-\varrho+1)\left({ }^{c} \mathscr{D}_{\tau_{1}^{+}}^{\varrho^{-1,9}} \mathcal{U}\right)\left(\tau_{2}\right)}{2\left(\vartheta\left(\tau_{2}\right)-\vartheta\left(\tau_{1}\right)\right)^{n-\varrho+1}} .
$$

Adding (51) and (52), we get the first inequality (42).

Corollary 3. If we take $\varrho=\delta$ in (42), then we get the following inequality for generalized Caputo fractional derivative operator:

$$
\begin{aligned}
& \left(\frac{1}{n-\varrho+1}\right) \mathscr{U}^{(n)}\left(\frac{\tau_{1}+\tau_{2}}{2}\right) \\
& \leq \frac{\Gamma(n-\varrho+1)\left\{\left({ }^{c} \mathscr{D}_{\tau_{1}^{+}}^{\varrho-1, \vartheta} \mathcal{U}\right)\left(\tau_{2}\right)+\left({ }^{c} \mathscr{D}_{\tau_{2}^{-}}^{\varrho-1, \vartheta} \mathcal{U}\right)\left(\tau_{1}\right)\right\}}{2\left(\vartheta\left(\tau_{2}\right)-\vartheta\left(\tau_{1}\right)\right)^{n-\varrho+1}} \\
& \leq \frac{\left[\left(\tau_{2}-\tau_{1}\right)\left[\mathcal{U}^{(n)}\left(\tau_{2}\right) \vartheta\left(\tau_{2}\right)-\mathscr{U}^{(n)}\left(\tau_{1}\right) \vartheta\left(\tau_{1}\right)\right]-\left(\mathcal{U}^{(n)}\left(\tau_{2}\right)-\mathscr{U}^{(n)}\left(\tau_{1}\right)\right) \int_{\tau_{1}}^{\tau_{2}} \vartheta(\lambda) \mathrm{d} \lambda\right]}{\left(\vartheta\left(\tau_{2}\right)-\vartheta\left(\tau_{1}\right)\right)\left(\tau_{2}-\tau_{1}\right)} .
\end{aligned}
$$

\section{4. $\breve{C}$ eby $\breve{s}$ ev Type Inequalities for the Caputo Fractional Derivative in the $\mathbf{9}$-Hilfer Sense Operators}

In this section, we present several $\breve{C}$ eby $\breve{s}$ ev type inequalities for Caputo fractional derivative in the 9 -Hilfer sense operator defined in (15).

Theorem 4. For $n \in \mathbb{N}, \varrho \geq 1$, and let there be two absolutely continuous functions $\mathcal{U}$ and $\mathscr{V}$ which are synchronous on $[0, \infty)$. Also, assume that $\vartheta$ be differentiable and strictly increasing on $[0, \infty)$ with $\vartheta(0)=0$. Then, for all $\lambda>0$, we have

$$
\left({ }^{c} \mathscr{D}_{0^{+}}^{\varrho, \vartheta} \mathscr{U} \mathscr{V}\right)(\lambda) \geq \frac{\Gamma(n-\varrho+1)}{(\vartheta(\lambda))^{n-\varrho}}\left({ }^{c} \mathscr{D}_{0^{+}}^{\varrho, \vartheta} \mathscr{U}\right)(\lambda)\left({ }^{c} \mathscr{D}_{0^{+}}^{\varrho, \vartheta} \mathscr{V}\right)(\lambda) .
$$

Proof. Since $\mathcal{U}^{(n)}$ and $\mathscr{V}^{(n)}$ are synchronous on $[0, \infty)$, we have

$$
\begin{aligned}
& \mathscr{U}^{(n)}(r) \mathscr{V}^{(n)}(r)+\mathcal{U}^{(n)}(s) \mathscr{V}^{(n)}(s) \geq \mathcal{U}^{(n)}(r) \mathscr{V}^{(n)}(s) \\
& \quad+\mathcal{U}^{(n)}(s) \mathscr{V}^{(n)}(r) .
\end{aligned}
$$

If we multiply both sides of inequality (55) by 


$$
\frac{1}{\Gamma(n-\varrho)} \vartheta^{\prime}(r)(\vartheta(\lambda)-\vartheta(r))^{n-\varrho-1}, \quad \lambda \in \mathbb{R},
$$

results in

$$
\begin{aligned}
& \frac{1}{\Gamma(n-\varrho)} \vartheta^{\prime}(r)(\vartheta(\lambda)-\vartheta(r))^{n-\varrho-1} \mathcal{U}^{(n)}(r) \mathscr{V}^{(n)}(r) \\
& +\mathscr{U}^{(n)}(s) \mathscr{V}^{(n)}(s) \frac{1}{\Gamma(n-\varrho)} \vartheta^{\prime}(r)(\vartheta(\lambda)-\vartheta(r))^{n-\varrho-1} \\
& \geq \mathscr{V}^{(n)}(s) \frac{1}{\Gamma(n-\varrho)} \vartheta^{\prime}(r)(\vartheta(\lambda)-\vartheta(r))^{n-\varrho-1} \mathcal{U}^{(n)}(r) \\
& +\mathcal{U}^{(n)}(s) \frac{1}{\Gamma(n-\varrho)} \vartheta^{\prime}(r)(\vartheta(\lambda)-\vartheta(r))^{n-\varrho-1} \mathscr{V}^{(n)}(r) .
\end{aligned}
$$

Further integrating both sides with respect to $r$ over $(0, \lambda)$ gives

$$
\begin{aligned}
& \frac{1}{\Gamma(n-\varrho)} \int_{0}^{\lambda} \vartheta^{\prime}(r)(\vartheta(\lambda)-\vartheta(r))^{n-\varrho-1} \mathcal{U}^{(n)}(r) \mathscr{V}^{(n)}(r) \mathrm{d} r \\
& +\mathscr{U}^{(n)}(s) \mathscr{V}^{(n)}(s) \frac{1}{\Gamma(n-\varrho)} \int_{0}^{\lambda} \vartheta^{\prime}(r)(\vartheta(\lambda)-\vartheta(r))^{n-\varrho-1} \mathrm{~d} r \\
& \geq \mathscr{V}^{(n)}(s) \frac{1}{\Gamma(n-\varrho)} \int_{0}^{\lambda} \vartheta^{\prime}(r)(\vartheta(\lambda)-\vartheta(r))^{n-\varrho-1} \mathcal{U}^{(n)}(r) \mathrm{d} r \\
& +\mathscr{U}^{(n)}(s) \frac{1}{\Gamma(n-\varrho)} \int_{0}^{\lambda} \vartheta^{\prime}(r)(\vartheta(\lambda)-\vartheta(r))^{n-\varrho-1} \mathscr{V}^{(n)}(r) \mathrm{d} r
\end{aligned}
$$

Consequently, it follows that

$$
\begin{aligned}
& \left({ }^{c} \mathscr{D}_{0^{+}}^{\varrho, 9} \mathscr{U} \mathscr{V}\right)(\lambda)+\mathscr{U}^{(n)}(s) \mathscr{V}^{(n)}(s) \frac{1}{\Gamma(n-\varrho)} \\
& \int_{0}^{\lambda} \vartheta^{\prime}(r)(\vartheta(\lambda)-\vartheta(r))^{n-\varrho-1} \mathrm{~d} r \\
& \geq \mathscr{V}^{(n)}(s)\left({ }^{c} \mathscr{D}_{0^{+}}^{\mathrm{\rho} \vartheta} \mathscr{U}\right)(\lambda)+\mathscr{U}^{(n)}(s)\left({ }^{c} \mathscr{D}_{0^{+}}^{\varrho, \vartheta} \mathscr{V}\right)(\lambda), \\
& \left({ }^{c} \mathscr{D}_{0^{+}}^{\varrho, \vartheta} \mathscr{U} \mathscr{V}\right)(\lambda) \mathcal{U}^{(n)}(s) \mathscr{V}^{(n)}(s) \frac{(\vartheta(\lambda))^{n-\varrho}}{\Gamma(n-\varrho+1)} \\
& \geq \mathscr{V}^{(n)}(s)\left({ }^{c} \mathscr{D}_{0^{+}}^{\varrho, \vartheta} \mathscr{U}\right)(\lambda)+\mathscr{U}^{(n)}(s)\left({ }^{c} \mathscr{D}_{0^{+}}^{\varrho, \vartheta} \mathscr{V}\right)(\lambda),
\end{aligned}
$$

where

$$
\frac{1}{\Gamma(n-\varrho)} \int_{0}^{\lambda} \vartheta^{\prime}(r)(\vartheta(\lambda)-\vartheta(r))^{n-\varrho^{-1}} \mathrm{~d} r=\frac{(\vartheta(\lambda))^{n-\varrho}}{(n-\varrho) \Gamma(n-\varrho)}
$$

If we multiply both sides of inequality (60) by

$$
\frac{1}{\Gamma(n-\varrho)} \vartheta^{\prime}(s)(\vartheta(\lambda)-\vartheta(s))^{n-\varrho-1}, \quad \lambda \in \mathbb{R}
$$

we arrive at

$$
\begin{aligned}
& \left({ }^{c} \mathscr{D}_{0^{+}}^{\varrho, \vartheta} \mathscr{U V}\right)(\lambda) \frac{1}{\Gamma(n-\varrho)} \vartheta^{\prime}(s)(\vartheta(\lambda)-\vartheta(s))^{n-\varrho-1} \\
& +\frac{1}{\Gamma(n-\varrho)} \vartheta^{\prime}(s)(\vartheta(\lambda)-\vartheta(s))^{n-\varrho-1} \mathcal{U}^{(n)}(s) \mathscr{V}^{(n)}(s) \frac{(\vartheta(\lambda))^{n-\varrho}}{\Gamma(n-\varrho+1)} \\
& \geq\left({ }^{c} \mathscr{D}_{0^{+}}^{\varrho, \vartheta} \mathscr{U}\right)(\lambda) \frac{1}{\Gamma(n-\varrho)} \vartheta^{\prime}(s)(\vartheta(\lambda)-\vartheta(s))^{n-\varrho-1} \mathscr{V}^{(n)}(s) \\
& +\left({ }^{c} \mathscr{D}_{0^{+}}^{\varrho, \vartheta} \mathscr{V}\right)(\lambda) \frac{1}{\Gamma(n-\varrho)} \vartheta^{\prime}(s)(\vartheta(\lambda)-\vartheta(s))^{n-\varrho-1} \mathcal{U}^{(n)}(s) .
\end{aligned}
$$

Now, integrating over $(0, \lambda)$ reveals

$$
\begin{aligned}
& \left({ }^{c} \mathscr{D}_{0^{+}}^{\varrho, \vartheta} \mathscr{U V}\right)(\lambda) \frac{1}{\Gamma(n-\varrho)} \int_{0}^{\lambda} \vartheta^{\prime}(s)(\vartheta(\lambda)-\vartheta(s))^{n-\varrho^{-1} \mathrm{~d} s} \\
& +\frac{1}{\Gamma(n-\varrho)} \int_{0}^{\lambda} \vartheta^{\prime}(s)(\vartheta(\lambda)-\vartheta(s))^{n-\varrho^{-1}} \mathscr{U}^{(n)}(s) \mathscr{V}^{(n)} \\
& \quad(s) \mathrm{d} s \frac{(\vartheta(\lambda))^{n-\varrho}}{\Gamma(n-\varrho+1)} \\
& \geq\left({ }^{c} \mathscr{D}_{0^{+}}^{\varrho, \vartheta} \mathcal{U}\right)(\lambda) \frac{1}{\Gamma(n-\varrho)} \int_{0}^{\lambda} \vartheta^{\prime}(s)(\vartheta(\lambda)-\vartheta(s))^{n-\varrho^{-1}} \mathscr{V}^{(n)}(s) \mathrm{d} s \\
& +\left({ }^{c} \mathscr{D}_{0^{+}}^{\varrho, \vartheta} \mathscr{V}\right)(\lambda) \frac{1}{\Gamma(n-\varrho)} \int_{0}^{\lambda} \vartheta^{\prime}(s)(\vartheta(\lambda)-\vartheta(s))^{n-\varrho^{-1}} \mathscr{U}^{(n)}(s) \mathrm{d} s .
\end{aligned}
$$

Therefore, we have

$$
\begin{aligned}
& \frac{(\vartheta(\lambda))^{n-\varrho}}{\Gamma(n-\varrho+1)}\left({ }^{c} \mathscr{D}_{0^{+}}^{\varrho, \vartheta} \mathscr{U} \mathscr{V}\right)(\lambda)+\left({ }^{c} \mathscr{D}_{0^{+}}^{\varrho, \vartheta} \mathscr{U} \mathscr{V}\right)(\lambda) \frac{(\vartheta(\lambda))^{n-\varrho}}{\Gamma(n-\varrho+1)} \\
& \geq\left({ }^{c} \mathscr{D}_{0^{+}}^{\varrho, \vartheta} \mathscr{U}\right)(\lambda)\left({ }^{c} \mathscr{D}_{0^{+}}^{\varrho, \vartheta} \mathscr{V}\right)(\lambda)+\left({ }^{c} \mathscr{D}_{0^{+}}^{\varrho, \vartheta} \mathscr{V}\right)(\lambda)\left({ }^{c} \mathscr{D}_{0^{+}}^{\varrho, \vartheta} \mathcal{U}\right)(\lambda) .
\end{aligned}
$$

The proof of Theorem 4 is complete.

Corollary 4. Setting $\varrho=1$, then under the assumptions of Theorem 4, we have

$$
\left({ }^{c} \mathscr{D}_{0^{+}}^{\vartheta} \mathcal{U} \mathscr{V}\right)(\lambda) \geq \frac{\Gamma(n)}{(\mathcal{V}(\lambda))^{n-1}}\left({ }^{c} \mathscr{D}_{0^{+}}^{\vartheta} \mathcal{U}\right)(\lambda)\left({ }^{c} \mathscr{D}_{0^{+}}^{\vartheta} \mathscr{V}\right)(\lambda) .
$$

Corollary 5. Setting $\vartheta(\lambda)=\lambda$, then under the assumption of Theorem 4, we have a new result for the Caputo fractional derivative operator:

$$
\left({ }^{c} \mathscr{D}_{0^{+}}^{\varrho} \mathscr{U} \mathscr{V}\right)(\lambda) \geq \frac{\Gamma(n-\varrho+1)}{(\lambda)^{n-\varrho}}\left({ }^{c} \mathscr{D}_{0^{+}}^{\varrho} \mathscr{U}\right)(\lambda)\left({ }^{c} \mathscr{D}_{0^{+}}^{\varrho} \mathscr{V}\right)(\lambda) .
$$

Theorem 5. For $n \in \mathbb{N}, \varrho, \delta \geq 1$, and let there be two absolutely continuous functions $\mathcal{U}$ and $\mathscr{V}$ which are synchronous on $[0, \infty)$. Also, assume that $\vartheta$ be differentiable and strictly 
increasing on $[0, \infty)$ with $\vartheta(0)=0$. Then, for all $\lambda>0$, we have

$$
\begin{aligned}
& \frac{\left({ }^{c} \mathscr{D}_{0^{+}}^{\mathrm{Q}, \vartheta} \mathscr{U V}\right)(\lambda)(\mathcal{V}(\lambda))^{n-\delta}}{\Gamma(n-\delta+1)}+\frac{(\mathcal{\vartheta}(\lambda))^{n-\rangle}\left({ }^{c} \mathscr{D}_{0^{+}}^{\delta, \vartheta} \mathscr{U} \mathscr{V}\right)(\lambda)}{\Gamma(n-\varrho+1)} \\
& \geq\left({ }^{c} \mathscr{D}_{0^{+}}^{\mathrm{e}, \vartheta} \mathscr{U}\right)(\lambda)\left({ }^{c} \mathscr{D}_{0^{+}}^{\delta, \vartheta} \mathscr{V}\right)(\lambda)+\left({ }^{c} \mathscr{D}_{0^{+}}^{\mathrm{e}, \vartheta} \mathscr{V}\right)(\lambda)\left({ }^{c} \mathscr{D}_{0^{+}}^{\delta, \vartheta} \mathscr{U}\right)(\lambda) .
\end{aligned}
$$

Proof. Using inequality (61) and multiplying both sides by

$$
\frac{1}{\Gamma(n-\delta)} \vartheta^{\prime}(s)(\vartheta(\lambda)-\vartheta(s))^{n-\delta-1}, \quad \lambda \in \mathbb{R},
$$

yields

$$
\begin{aligned}
& \left({ }^{c} \mathscr{D}_{0^{+}}^{\varrho, \vartheta} \mathscr{U V}\right)(\lambda) \frac{1}{\Gamma(n-\delta)} \mathcal{\vartheta}^{\prime}(s)(\vartheta(\lambda)-\vartheta(s))^{n-\delta-1} \\
& +\frac{1}{\Gamma(n-\delta)} \mathcal{\vartheta}^{\prime}(s)(\vartheta(\lambda)-\vartheta(s))^{n-\delta-1} \mathscr{U}^{(n)}(s) \mathscr{V}^{(n)}(s) \frac{(\vartheta(\lambda))^{n-\varrho}}{\Gamma(n-\varrho+1)} \\
& \geq\left({ }^{c} \mathscr{D}_{0^{+}}^{\varrho, \vartheta} \mathscr{U}\right)(\lambda) \frac{1}{\Gamma(n-\delta)} \vartheta^{\prime}(s)(\vartheta(\lambda)-\vartheta(s))^{n-\delta-1} \mathscr{V}^{(n)}(s) \\
& +\left({ }^{c} \mathscr{D}_{0^{+}}^{\varrho, \vartheta} \mathscr{V}\right)(\lambda) \frac{1}{\Gamma(n-\delta)} \vartheta^{\prime}(s)(\vartheta(\lambda)-\vartheta(s))^{n-\delta-1} \mathcal{U}^{(n)}(s) .
\end{aligned}
$$

Furthermore, integrating both sides with respect to $s$ over $(0, \lambda)$ leads to

$$
\begin{aligned}
& \frac{\left({ }^{c} \mathscr{D}_{0^{+}}^{\varrho, \vartheta} \mathscr{U}\right)(\lambda)}{\Gamma(n-\delta)} \int_{0}^{\lambda} \vartheta^{\prime}(s)(\vartheta(\lambda)-\vartheta(s))^{n-\delta-1} \mathrm{~d} s \\
& +\frac{(\vartheta(\lambda))^{n-\varrho}}{\Gamma(n-\varrho+1)} \frac{1}{\Gamma(n-\delta)} \int_{0}^{\lambda} \vartheta^{\prime}(s)(\vartheta(\lambda)-\vartheta(s))^{n-\delta-1} \mathcal{U}^{(n)} \\
& \geq \frac{(s) \mathscr{V}^{(n)}(s) \mathrm{d} s}{\Gamma(n-\delta)} \int_{0}^{\lambda} \mathscr{\vartheta}^{\varrho}(s)(\vartheta(\lambda)-\vartheta(s))^{n-\delta-1} \mathscr{V}^{(n)}(s) \mathrm{d} s \\
& +\frac{\left({ }^{c} \mathscr{D}_{0^{+}}^{\mathrm{Q}, \vartheta} \mathscr{V}\right)(\lambda)}{\Gamma(n-\delta)} \int_{0}^{\lambda} \vartheta^{\prime}(s)(\vartheta(\lambda)-\vartheta(s))^{n-\delta-1} \mathcal{U}^{(n)}(s) \mathrm{d} s .
\end{aligned}
$$

Therefore, we have

$$
\begin{aligned}
& \frac{\left({ }^{c} \mathscr{D}_{0^{+}}^{\varrho, \vartheta} \mathscr{U V}\right)(\lambda)(\vartheta(\lambda))^{n-\delta}}{(n-\delta+1)}+\frac{(\vartheta(\lambda))^{n-\rangle}\left({ }^{c} \mathscr{D}_{0^{+}}^{\delta, \vartheta} \mathscr{U} \mathscr{V}\right)(\lambda)}{\Gamma(n-\varrho+1)} \\
& \geq\left({ }^{c} \mathscr{D}_{0^{+}}^{\varrho, 9} \mathscr{U}\right)(\lambda)\left({ }^{c} \mathscr{D}_{0^{+}}^{\delta, \vartheta} \mathscr{V}\right)(\lambda)+\left({ }^{c} \mathscr{D}_{0^{+}}^{\varrho, 9} \mathscr{V}\right)(\lambda)\left({ }^{c} \mathscr{D}_{0^{+}}^{\delta, \vartheta} \mathscr{U}\right)(\lambda) .
\end{aligned}
$$

Hence, this completes the proof.

Remark. Applying Theorem 5 to $\varrho=\delta$ results in Theorem 4 .
Corollary 6. Setting $\varrho=\delta=1$, then under the assumptions of Theorem 5, we have

$$
\left({ }^{c} \mathscr{D}_{0^{+}}^{\vartheta} \mathscr{U} \mathscr{V}\right)(\lambda) \geq \frac{\Gamma(n)}{(\mathcal{V}(\lambda))^{n-1}}\left({ }^{c} \mathscr{D}_{0^{+}}^{\vartheta} \mathscr{U}\right)(\lambda)\left({ }^{c} \mathscr{D}_{0^{+}}^{\vartheta} \mathscr{V}\right)(\lambda) .
$$

Corollary 7. Setting $\vartheta(\lambda)=\lambda$, then under the assumption of Theorem 5, we have inequality for Caputo fractional derivative:

$$
\begin{aligned}
& \frac{\lambda^{n-\delta}\left({ }^{c} \mathscr{D}_{0^{+}}^{\varrho} \mathscr{U V}\right)(\lambda)}{\Gamma(n-\delta+1)}+\frac{\lambda^{n-\rangle}\left({ }^{c} \mathscr{D}_{0^{+}}^{\delta} \mathscr{U} \mathscr{V}\right)(\lambda)}{\Gamma(n-\varrho+1)} \\
& \geq\left({ }^{c} \mathscr{D}_{0^{+}}^{\varrho} \mathscr{U}\right)(\lambda)\left({ }^{c} \mathscr{D}_{0^{+}}^{\delta} \mathscr{V}\right)(\lambda)+\left({ }^{c} \mathscr{D}_{0^{+}}^{\varrho} \mathscr{V}\right)(\lambda)\left({ }^{c} \mathscr{D}_{0^{+}}^{\delta} \mathscr{U}\right)(\lambda) .
\end{aligned}
$$

Theorem 6. For $n \in \mathbb{N}, \varrho \geq 1$, and let $\mathcal{U}_{j}^{(n)}$ for $1 \leq j \leq \kappa$ be real-valued increasing functions defined on $[0, \infty)$. Also, assume that $\vartheta$ be differentiable and strictly increasing on $[0, \infty)$ with $\vartheta(0)=0$. Then, for all $\lambda>0$, we have

$$
\left({ }^{c} \mathscr{D}_{0^{+}}^{\varrho, \vartheta} \prod_{j=1}^{\kappa} \mathcal{U}_{j}\right)(\lambda) \geq\left[\frac{\Gamma(n-\varrho+1)}{(\vartheta(\lambda))^{n-\rangle}}\right]^{\kappa-1} \prod_{j=1}^{\kappa}\left({ }^{c} \mathscr{D}_{0^{+}}^{\varrho, \vartheta} \mathcal{U}_{j}\right)(\lambda) .
$$

Proof. To prove the present theorem, we use mathematical induction on $\kappa \in \mathbb{N}$. Clearly, the case $\kappa=1$ of (75) holds.

For $\kappa=2$, since $\mathscr{U}_{1}^{(n)}, \mathcal{U}_{2}^{(n)}$ are increasing, we have

$$
\left\langle\mathcal{U}_{1}^{(n)}(\lambda)-\mathcal{U}_{1}^{(n)}(\omega), \mathcal{U}_{2}^{(n)}(\lambda)-\mathcal{U}_{2}^{(n)}(\omega)\right\rangle \geq 0 .
$$

Now, the left part of inequality (75) for $\kappa=2$ is the same as that of Theorem 4 .

Suppose that inequality (82) holds for some $\kappa \geq 3$. We observe that, since $\mathscr{U}_{j}^{(n)}$ is increasing, $\mathcal{U}=\prod_{j=1}^{\kappa} \mathcal{U}_{j}^{(n)}$ is increasing. Let $\mathscr{V}=\mathscr{U}_{\kappa+1}^{(n)}$. Then, applying the case $\kappa=2$ to the function $\mathscr{U}$ and $\mathscr{V}$ produces

$$
\begin{aligned}
& \left({ }^{c} \mathscr{D}_{0^{+}}^{\varrho, 9} \prod_{j=1}^{\kappa} \mathcal{U}_{j} \mathcal{U}_{\kappa+1}\right)(\lambda) \geq\left[\frac{\Gamma(n-\varrho+1)}{(\vartheta(\lambda))^{n-\varrho}}\right] \\
& \left({ }^{c} \mathscr{D}_{0^{+}}^{\varrho, \vartheta} \prod_{j=1}^{\kappa} \mathcal{U}_{j}\right)\left({ }^{c} \mathscr{D}_{0^{+}}^{\varrho, \vartheta} \mathcal{U}_{\kappa+1}\right)(\lambda) \\
& \geq\left[\frac{\Gamma(n-\varrho+1)}{(\vartheta(\lambda))^{n-\varrho}}\right]^{\kappa} \prod_{j=1}^{\kappa+1}\left({ }^{c} \mathscr{D}_{0^{+}}^{\varrho, \kappa} \mathcal{U}_{j}\right)(\lambda),
\end{aligned}
$$

in which the induction hypothesis for $\kappa$ is used inside the deduction of second inequality. The proof of Theorem 6 is complete.

Corollary 8. Let $\mathcal{U}_{j}^{(n)}$ for $1 \leq j \leq \kappa$ be real-valued increasing functions defined on $[0, \infty)$. For $\mathrm{\varrho}>0$, we have 


$$
\left({ }^{c} \mathscr{D}_{0^{+}}^{9} \prod_{j=1}^{\kappa} \mathcal{U}_{j}\right)(\lambda) \geq\left[\frac{\Gamma(n)}{(\mathcal{(}(\lambda))^{n-1}}\right]^{\kappa-1} \prod_{j=1}^{\kappa}\left(\mathscr{D}_{0^{+}}^{9} \mathcal{U}_{j}\right)(\lambda) .
$$

Proof. This follows from taking $\varrho=1$ in Theorem 6 .

Corollary 9. If we choose $9(\lambda)=\lambda$, then under the assumptions of Theorem 6, we have a new result for Caputo fractional derivative operator:

$$
\left({ }^{c} \mathscr{D}_{0^{+}}^{\varrho} \prod_{j=1}^{\kappa} \mathcal{U}_{j}\right)(\lambda) \geq\left[\frac{\Gamma(n-\varrho+1)}{\lambda^{n-\varrho}}\right]^{\kappa-1} \prod_{j=1}^{\kappa}\left(\mathscr{D}_{0^{+}}^{\varrho} \mathscr{U}_{j}\right)(\lambda) .
$$

Theorem 7. For $n \in \mathbb{N} \varrho \geq 1$, let $\mathcal{U}, \mathscr{V}$ be two absolutely continuous mappings on $[0, \infty)$ such that $\mathcal{U}^{(n)}$ is increasing, $\mathscr{V}^{(n)}$ is differentiable, and $\mathscr{V}^{(n+1)}$ is a lower bound $c=\inf _{\mu \in[0, \infty)} \mathscr{V}^{(n+1)}(\mu)$. Also, assume that $\vartheta$ be differentiable and strictly increasing on $[0, \infty)$ with $\vartheta(0)=0$. Then, for all $\lambda>0$, we have

$$
\begin{gathered}
\left({ }^{c} \mathscr{D}_{0^{+}}^{\mathrm{e}, \vartheta} \mathscr{U V}\right)(\lambda) \geq \frac{\Gamma(n-\varrho+1)}{(\vartheta(\lambda))^{n-\varrho}}\left({ }^{c} \mathscr{D}_{0^{+}}^{\mathrm{e}, 9} \mathscr{U}\right)(\lambda)\left({ }^{c} \mathscr{D}_{0^{+}}^{\mathrm{e}, \vartheta} \mathscr{V}\right)(\lambda) \\
-\frac{\theta \lambda(\vartheta(\lambda))^{n-\varrho}}{\Gamma(n-\varrho+1)}\left({ }^{c} \mathscr{D}_{0^{+}}^{\mathrm{e}, \vartheta} \mathscr{U}\right)(\lambda)+\theta\left({ }^{c} \mathscr{D}_{0^{+}}^{\mathrm{e}, \mathcal{G}} \mathscr{I} \mathcal{U}\right)(\lambda),
\end{gathered}
$$

where $\mathscr{I}(\lambda)$ is the identity function.

Proof. Let $h(\lambda)=\mathscr{V}(\lambda)-\theta \lambda$. We shall show that $h$ is differentiable and increasing on $[0, \infty)$. As we did in the proof of Theorem 6, for clarity, let $\Upsilon(\lambda)=\theta \lambda$, and we find

$$
\begin{aligned}
& \left({ }^{c} \mathscr{D}_{0^{+}}^{\varrho, \vartheta} \mathscr{U}(\mathscr{V}-\Upsilon)\right)(\lambda) \geq \frac{\Gamma(n-\varrho+1)}{(\vartheta(\lambda))^{n-\varrho}}\left({ }^{c} \mathscr{D}_{0^{+}}^{\varrho, \vartheta} \mathcal{U}\right)(\lambda) \\
& \left({ }^{c} \mathscr{D}_{0^{+}}^{\varrho, \vartheta}(\mathscr{V}-\Upsilon)\right)(\lambda) \\
& =\frac{\Gamma(n-\varrho+1)}{(\vartheta(\lambda))^{n-\varrho}}\left({ }^{c} \mathscr{D}_{0^{+}}^{\varrho, \vartheta} \mathcal{U}\right)(\lambda)\left({ }^{c} \mathscr{D}_{0^{+}}^{\varrho, \vartheta} \mathscr{V}\right)(\lambda) \\
& -\frac{\Gamma(n-\varrho+1)}{(\vartheta(\lambda))^{n-\varrho}}\left({ }^{c} \mathscr{D}_{0^{+}}^{\varrho, \vartheta} \mathscr{U}\right)(\lambda)\left({ }^{c} \mathscr{D}_{0^{+}}^{\varrho, \vartheta} \Upsilon\right)(\lambda),
\end{aligned}
$$

where

$$
\begin{gathered}
\left({ }^{c} \mathscr{D}_{0^{+}}^{\mathrm{e}, 9} \mathcal{U}(\mathscr{V}-\Upsilon)\right)(\lambda)=\left({ }^{c} \mathscr{D}_{0^{+}}^{\mathrm{\rho}, 9} \mathcal{U V}\right)(\lambda)-\theta\left({ }^{c} \mathscr{D}_{0^{+}}^{\mathrm{e}, 9} \mathscr{I} \mathcal{U}\right)(\lambda) \\
\left({ }^{c} \mathscr{D}_{0^{+}}^{\mathrm{e}, 9} \Upsilon\right)(\lambda)=\frac{\theta \lambda(\vartheta(\lambda))^{n-\varrho}}{\Gamma(n-\varrho+1)}
\end{gathered}
$$

Substituting (82) and (83) into (81) leads to the desired results.
Corollary 10. If we choose $\mathrm{Q}=1$, then under the assumption of Theorem 7, we have

$$
\begin{gathered}
\left({ }^{c} \mathscr{D}_{0^{+}}^{\vartheta} \mathscr{U} \mathscr{V}\right)(\lambda) \geq \frac{\Gamma(n)}{(\vartheta(\lambda))^{n-1}}\left({ }^{c} \mathscr{D}_{0^{+}}^{\vartheta} \mathcal{U}\right)(\lambda)\left({ }^{c} \mathscr{D}_{0^{+}}^{\vartheta} \mathscr{V}\right)(\lambda) \\
-\frac{\theta \lambda(\vartheta(\lambda))^{n}}{\Gamma(n)}\left({ }^{c} \mathscr{D}_{0^{+}}^{\vartheta} \mathcal{U}\right)(\lambda)+\theta\left({ }^{c} \mathscr{D}_{0^{+}}^{\vartheta} \mathscr{I} \mathscr{U}\right)(\lambda),
\end{gathered}
$$

where $\mathscr{I}(\lambda)$ is the identity function.

Corollary 11. If we choose $\vartheta(\lambda)=\lambda$, then under the assumption of Theorem 7 , we have a new result for Caputo fractional derivative operator:

$$
\begin{gathered}
\left({ }^{c} \mathscr{D}_{0^{+}}^{\varrho} \mathscr{U V}\right)(\lambda) \geq \frac{\Gamma(n-\varrho+1)}{\lambda^{n-\rangle}}\left({ }^{c} \mathscr{D}_{0^{+}}^{\varrho} \mathscr{U}\right)(\lambda)\left({ }^{c} \mathscr{D}_{0^{+}}^{\varrho} \mathscr{V}\right)(\lambda) \\
-\frac{\theta \lambda^{n-\varrho+1}}{\Gamma(n-\varrho+1)}\left({ }^{c} \mathscr{D}_{0^{+}}^{\varrho} \mathscr{U}\right)(\lambda)+\theta\left({ }^{c} \mathscr{D}_{0^{+}}^{\varrho} \mathscr{J} \mathscr{U}\right)(\lambda),
\end{gathered}
$$

where $\mathscr{I}(\lambda)$ is the identity function.

\section{Grüss Type Inequalities for the Caputo Fractional Derivative in the 9 -Hilfer Sense Operators}

In this section, we prove some Grüss type inequalities involving the Caputo fractional derivative in the 9 -Hifer sense operator defined in (15).

Theorem 8. For $n \in \mathbb{N}, \varrho, \delta>0$, and let there be an absolutely continuous function $\mathcal{U}$ defined on $[0, \infty)$. Also, assume that $\vartheta$ be differentiable and strictly increasing on $[0, \infty)$ with $\vartheta(0)=0$. Suppose that there exist two integrable functions $\Upsilon_{1}^{(n)}, \Upsilon_{2}^{(n)}$ on $[0, \infty)$ such that

$$
\Upsilon_{1}^{(n)}(\lambda) \leq \mathscr{U}^{(n)}(\lambda) \leq \Upsilon_{2}^{(n)}(\lambda), \quad \forall \lambda \in[0, \infty) .
$$

Then, we obtain the following inequality for the generalized Caputo fractional integral operator:

$$
\begin{aligned}
& { }^{c} \mathscr{D}_{0^{+}}^{\vartheta, \delta} \Upsilon_{1}(\lambda)^{c} \mathscr{D}_{0^{+}}^{\vartheta,\rangle} \mathcal{U}(\lambda)+{ }^{c} \mathscr{D}_{0^{+}}^{\vartheta, \rho} \Upsilon_{2}(\lambda)^{c} \mathscr{D}_{0^{+}}^{\vartheta, \delta} \mathcal{U}(\lambda) \\
& \geq^{c} \mathscr{D}_{0^{+}}^{\vartheta, \rho} \Upsilon_{2}(\lambda)^{c} \mathscr{D}_{0^{+}}^{\vartheta, \delta} \Upsilon_{1}(\lambda)+{ }^{c} \mathscr{D}_{0^{+}}^{\vartheta, \rho} \mathcal{U}(\lambda)^{c} \mathscr{D}_{0^{+}}^{\vartheta, \delta} \mathcal{U}(\lambda) .
\end{aligned}
$$

Proof. From (86), for all $r \geq 0, s \geq 0$, we have

$$
\begin{aligned}
& \left(\Upsilon_{2}^{(n)}(r)-\mathcal{U}^{(n)}(r)\right)\left(\mathcal{U}^{(n)}(s)-\Upsilon_{1}^{(n)}(s)\right) \geq 0, \\
& \Upsilon_{2}^{(n)}(r) \mathcal{U}^{(n)}(s)+\Upsilon_{1}^{(n)}(s) \mathcal{U}^{(n)}(r) \Upsilon_{1}^{(n)}(s) \Upsilon_{2}^{(n)}(r) \\
& \quad+\mathcal{U}^{(n)}(r) \mathcal{U}^{(n)}(s) .
\end{aligned}
$$

If we multiply both sides of (86) by $\left((\vartheta(\lambda)-\vartheta(r))^{n-\varrho^{-1}} \vartheta^{\prime}(r)\right) / \Gamma(n-\varrho)$ and integrating with respect to $r$ on $(0, \lambda)$, we obtain 


$$
\begin{aligned}
& \mathcal{U}^{(n)}(s) \frac{1}{\Gamma(n-\varrho)} \int_{0}^{\lambda}(\vartheta(\lambda)-\vartheta(r))^{n-\varrho-1} \vartheta^{\prime}(r) \Upsilon_{2}^{(n)}(r) \mathrm{d} r \\
& +\Upsilon_{1}^{(n)}(s) \frac{1}{\Gamma(n-\varrho)} \int_{0}^{\lambda}(\vartheta(\lambda)-\vartheta(r))^{n-\varrho-1} \vartheta^{\prime}(r) \mathcal{U}^{(n)}(r) \mathrm{d} r \\
& \geq \Upsilon_{1}^{(n)}(s) \frac{1}{\Gamma(n-\varrho)} \int_{0}^{\lambda} \int_{0}^{\lambda}(\vartheta(\lambda)-\vartheta(r))^{n-\varrho-1} \mathcal{\vartheta}^{\prime}(r) \Upsilon_{2}^{(n)}(r) \mathrm{d} r+ \\
& \mathcal{U}^{(n)}(s) \frac{1}{\Gamma(n-\varrho)} \int_{0}^{\lambda}(\mathcal{\vartheta}(\lambda)-\vartheta(r))^{n-\varrho-1} \mathcal{\vartheta}^{\prime}(r) \mathcal{U}^{(n)}(r) \mathrm{d} r,
\end{aligned}
$$

which can be written as follows:

$$
\begin{aligned}
& \mathscr{U}^{(n)}(s)^{c} \mathscr{D}_{0^{+}}^{9, \mathrm{e}} \Upsilon_{2}(\lambda)+\Upsilon_{1}^{(n)}(s)^{c} \mathscr{D}_{0^{+}}^{9, \mathrm{e}} \mathcal{U}(\lambda) \geq \Upsilon_{1}^{(n)}(s)^{c} \mathscr{D}_{0^{+}}^{9, \mathrm{e}} \Upsilon_{2} \\
& (\lambda)+\mathscr{U}^{(n)}(s)^{c} \mathscr{D}_{0^{+}}^{9, \mathrm{e}} \mathcal{U}(\lambda) .
\end{aligned}
$$

If we multiply both sides of (90) bs $\left((\vartheta(\lambda)-\vartheta(s))^{n-\delta-1} \mathcal{\vartheta}^{\prime}(s)\right) / \Gamma(n-\delta)$ and integrating with respect to $s$ on $(0, \lambda)$, we obtain

$$
\begin{aligned}
& { }^{c} \mathscr{D}_{0^{+}}^{9, \delta} \Upsilon_{1}(\lambda)^{c} \mathscr{D}_{0^{+}}^{9, \rho} \mathscr{U}(\lambda)+{ }^{c} \mathscr{D}_{0^{+}}^{9, \rho} \Upsilon_{2}(\lambda)^{c} \mathscr{D}_{0^{+}}^{9, \delta} \mathscr{U}(\lambda) \\
& \geq^{c} \mathscr{D}_{0^{+}}^{9, \mathrm{e}} \Upsilon_{2}(\lambda)^{c} \mathscr{D}_{0^{+}}^{9, \delta} \Upsilon_{1}(\lambda)+{ }^{c} \mathscr{D}_{0^{+}}^{9, \mathrm{e}} \mathscr{U}(\lambda)^{c} \mathscr{D}_{0^{+}}^{9, \delta} \mathscr{U}(\lambda) .
\end{aligned}
$$

Hence, this completes the proof.
Corollary 12. Let $9(\lambda)=\lambda$ in Theorem 8 ; then, we have the inequality for Caputo-type fractional derivative operator:

$$
\begin{aligned}
& { }^{c} \mathscr{D}_{0^{+}}^{\delta} \Upsilon_{1}(\lambda)^{c} \mathscr{D}_{0^{+}}^{\varrho} \mathcal{U}(\lambda)+{ }^{c} \mathscr{D}_{0^{+}}^{\varrho} \Upsilon_{2}(\lambda)^{c} \mathscr{D}_{0^{+}}^{\delta} \mathcal{U}(\lambda) \\
& \geq{ }^{c} \mathscr{D}_{0^{+}}^{\varrho} \Upsilon_{2}(\lambda)^{c} \mathscr{D}_{0^{+}}^{\delta} \Upsilon_{1}(\lambda)+{ }^{c} \mathscr{D}_{0^{+}}^{\varrho} \mathcal{U}(\lambda)^{c} \mathscr{D}_{0^{+}}^{\delta} \mathcal{U}(\lambda) .
\end{aligned}
$$

Corollary 13. Let $\mathcal{U}$ be an absolutely continuous on $[0, \infty)$. Suppose that $q \leq \mathscr{U}^{(n)}(\lambda) \leq Q$, for all $\lambda \in[0, \infty)$ and $q, Q \in \mathscr{R}$. Then, for $\lambda>0, \varrho>0, \delta>0$, we have

$$
\begin{aligned}
& q \frac{\vartheta^{n-\delta}(\lambda)}{\Gamma(n-\delta+1)}\left({ }^{c} \mathscr{D}_{0^{+}}^{\vartheta, \varrho} \mathcal{U}(\lambda)\right)+Q \frac{\vartheta^{n-\varrho}(\lambda)}{\Gamma(n-\varrho+1)}\left({ }^{c} \mathscr{D}_{0^{+}}^{\vartheta, \delta} \mathcal{U}(\lambda)\right) \\
& \geq q Q \frac{\vartheta^{n-\delta}(\lambda)}{\Gamma(n-\delta+1)} \frac{\vartheta^{n->}(\lambda)}{\Gamma(n-\varrho+1)}+{ }^{c} \mathscr{D}_{0^{+}}^{\vartheta, \varrho} \mathcal{U}(\lambda)^{c} \mathscr{D}_{0^{+}}^{\vartheta, \delta} \mathcal{U}(\lambda) .
\end{aligned}
$$

Theorem 9. For $n \in \mathbb{N}, \varrho, \delta>0$, and let there be an absolutely continuous function $\mathcal{U}$ defined on $[0, \infty)$. Also, assume that $\vartheta$ be differentiable and strictly increasing on $[0, \infty)$ with $\vartheta(0)=0$. Suppose that (86) holds, and moreover assume that there exist $\varphi_{1}^{(n)}$ and $\varphi_{2}^{(n)}$ integrable functions on $[0, \infty)$ such that

$$
\varphi_{1}^{(n)}(\lambda) \leq \mathscr{V}^{(n)}(\lambda) \leq \varphi_{2}^{(n)}(\lambda), \quad \forall \lambda \in[0, \infty) .
$$

Then, the following inequalities hold for generalized Caputo fractional derivative operator:
(a) ${ }^{c} \mathscr{D}_{0^{+}}^{9, \delta} \varphi_{1}(\lambda)^{c} \mathscr{D}_{0^{+}}^{9, \rho} \mathscr{U}(\lambda)+{ }^{c} \mathscr{D}_{0^{+}}^{9, \mathrm{e}} \Upsilon_{2}(\lambda)^{c} \mathscr{D}_{0^{+}}^{9, \delta} \mathscr{V}(\lambda) \geq^{c} \mathscr{D}_{0^{+}}^{9, \delta} \varphi_{1}(\lambda)^{c} \mathscr{D}_{0^{+}}^{9, \mathrm{e}} \Upsilon_{2}(\lambda)+{ }^{c} \mathscr{D}_{0^{+}}^{9, \mathrm{e}} \mathscr{U}(\lambda)^{c} \mathscr{D}_{0^{+}}^{9, \delta} \mathscr{V}(\lambda)$,
(b) ${ }^{c} \mathscr{D}_{0^{+}}^{9, \delta} \Upsilon_{1}(\lambda)^{c} \mathscr{D}_{0^{+}}^{9, \rho} \mathscr{V}(\lambda)+{ }^{c} \mathscr{D}_{0^{+}}^{\vartheta, \rho} \varphi_{2}(\lambda)^{c} \mathscr{D}_{0^{+}}^{9, \delta} \mathscr{U}(\lambda) \geq{ }^{c} \mathscr{D}_{0^{+}}^{\vartheta, \delta} \Upsilon_{1}(\lambda)^{c} \mathscr{D}_{0^{+}}^{9, \rho} \varphi_{2}(\lambda)+{ }^{c} \mathscr{D}_{0^{+}}^{9, \delta} \mathscr{U}(\lambda)^{c} \mathscr{D}_{0^{+}}^{\vartheta, \rho} \mathscr{V}(\lambda)$,
(c) ${ }^{c} \mathscr{D}_{0^{+}}^{9, \rho} \Upsilon_{2}(\lambda)^{c} \mathscr{D}_{0^{+}}^{9, \delta} \varphi_{2}(\lambda)+{ }^{c} \mathscr{D}_{0^{+}}^{9, \rho} \mathcal{U}(\lambda)^{c} \mathscr{D}_{0^{+}}^{9, \delta} \mathscr{V}(\lambda) \geq{ }^{c} \mathscr{D}_{0^{+}}^{9, \rho} \Upsilon_{2}(\lambda)^{c} \mathscr{D}_{0^{+}}^{9, \delta} \mathscr{V}(\lambda)+{ }^{c} \mathscr{D}_{0^{+}}^{9, \delta} \varphi_{2}(\lambda)^{c} \mathscr{D}_{0^{+}}^{9, \rho} \mathscr{U}(\lambda)$,
(d) ${ }^{c} \mathscr{D}_{0^{+}}^{9, \rho} \Upsilon_{1}(\lambda)^{c} \mathscr{D}_{0^{+}}^{9, \delta} \varphi_{1}(\lambda)+{ }^{c} \mathscr{D}_{0^{+}}^{9, \rho} \mathscr{U}(\lambda)^{c} \mathscr{D}_{0^{+}}^{9, \delta} \mathscr{V}(\lambda) \geq^{c} \mathscr{D}_{0^{+}}^{9, \rho} \Upsilon_{1}(\lambda)^{c} \mathscr{D}_{0^{+}}^{9, \delta} \mathscr{V}(\lambda)+{ }^{c} \mathscr{D}_{0^{+}}^{9, \delta} \varphi_{1}(\lambda)^{c} \mathscr{D}_{0^{+}}^{9, \rho} \mathscr{U}(\lambda)$.

Proof. From (86) and (94) for all $r \geq 0, s \geq 0$, we have

$$
\left(\Upsilon_{2}^{(n)}(r)-\mathscr{U}^{(n)}(r)\right)\left(\mathscr{V}^{(n)}(s)-\varphi_{1}^{(n)}(s)\right) \geq 0,
$$

then

$$
\begin{aligned}
& \Upsilon_{2}^{(n)}(r) \mathscr{V}^{(n)}(s)+\varphi_{1}^{(n)}(s) \mathcal{U}^{(n)}(r) \geq \varphi_{1}^{(n)}(s) \Upsilon_{2}^{(n)}(r) \\
& +\mathcal{U}^{(n)}(r) \mathscr{V}^{(n)}(s) .
\end{aligned}
$$

If we multiply both sides of (97) by $\left((\vartheta(\lambda)-\vartheta(r))^{n-\varrho^{-1}} \mathcal{\vartheta}^{\prime}(r)\right) / \Gamma(n-\varrho)$, and integrating with respect to $r$ on $(0, \lambda)$, we have

$$
\begin{aligned}
& \mathscr{V}^{(n)}(s) \frac{1}{\Gamma(n-\varrho)} \int_{0}^{\lambda}(\vartheta(\lambda)-\vartheta(r))^{n-\varrho-1} \vartheta^{\prime}(r) \Upsilon_{2}^{(n)}(r) \mathrm{d} r \\
& +\varphi_{1}^{(n)}(s) \frac{1}{\Gamma(n-\varrho)} \int_{0}^{\lambda}(\vartheta(\lambda)-\vartheta(r))^{n-\varrho-1} \vartheta^{\prime}(r) \mathcal{U}^{(n)}(r) \mathrm{d} r \\
& \geq \varphi_{1}^{(n)}(s) \frac{1}{\Gamma(n-\varrho)} \int_{0}^{\lambda}(\vartheta(\lambda)-\vartheta(r))^{n-\varrho-1} \vartheta^{\prime}(r) \Upsilon_{2}^{(n)}(r) \mathrm{d} r \\
& +\mathscr{V}^{(n)}(s) \frac{1}{\Gamma(n-\varrho)} \int_{0}^{\lambda}(\vartheta(\lambda)-\vartheta(r))^{n-\varrho-1} \vartheta^{\prime}(r) \mathcal{U}^{(n)}(r) \mathrm{d} r .
\end{aligned}
$$


It can be written as

$$
\begin{aligned}
& \mathscr{V}^{(n)}(s)^{c} \mathscr{D}_{0^{+}}^{9, \mathrm{e}} \Upsilon_{2}(\lambda)+\varphi_{1}^{(n)}(s)^{c} \mathscr{D}_{0^{+}}^{9, \mathrm{e}} \mathscr{U}(\lambda) \\
& \geq \varphi_{1}^{(n)}(s)^{c} \mathscr{D}_{0^{+}}^{9, \mathrm{e}} \Upsilon_{2}(\lambda)+\mathscr{V}^{(n)}(s)^{c} \mathscr{D}_{0^{+}}^{9, \mathrm{e}} \mathscr{U}(\lambda) .
\end{aligned}
$$

If we multiply both sides of (99) by $\left((\vartheta(\lambda)-\vartheta(s))^{n-\delta-1} \vartheta^{\prime}(s)\right) / \Gamma(n-\delta)$ and integrating with respect to $s$ on $(0, \lambda)$, we obtain

$$
\begin{aligned}
& { }^{c} \mathscr{D}_{0^{+}}^{9, \delta} \varphi_{1}(\lambda)^{c} \mathscr{D}_{0^{+}}^{9, \rho} \mathscr{U}(\lambda)+{ }^{c} \mathscr{D}_{0^{+}}^{9, \rho} \Upsilon_{2}(\lambda)^{c} \mathscr{D}_{0^{+}}^{9, \delta} \mathscr{V}(\lambda) \\
& \geq^{c} \mathscr{D}_{0^{+}}^{9, \delta} \varphi_{1}(\lambda)^{c} \mathscr{D}_{0^{+}}^{9, \rho} \Upsilon_{2}(\lambda)+{ }^{c} \mathscr{D}_{0^{+}}^{9, \rho} \mathscr{U}(\lambda)^{c} \mathscr{D}_{0^{+}}^{9, \delta} \mathscr{V}(\lambda) .
\end{aligned}
$$

This proves (a).

To prove $(\mathrm{b})-(\mathrm{d})$, we use the following inequalities:

(b) $\left(\varphi_{2}^{(n)}(r)-\mathscr{V}^{(n)}(r)\right)\left(\mathscr{U}^{(n)}(s) \Upsilon_{1}^{(n)}(s)\right) \geq 0$,

(c) $\left(\Upsilon_{2}^{(n)}(r)-\mathscr{U}^{(n)}(r)\right)\left(\mathscr{V}^{(n)}(s)-\varphi_{2}^{(n)}(s)\right) \leq 0$,

(d) $\left(\Upsilon_{1}^{(n)}(r)-\mathscr{U}^{(n)}(r)\right)\left(\mathscr{V}^{(n)}(s)-\varphi_{1}^{(n)}(s)\right) \leq 0$.

The following inequalities are special cases of Theorem 9.

Corollary 14. Let $\mathscr{U}$ and $\mathscr{V}$ be two absolutely continuous on $[0, \infty), \lambda, \varrho, \delta>0$. Suppose that there exist real constants $q, Q, p, P$ such that

$$
\begin{aligned}
& q \leq \mathscr{U}^{(n)}(\lambda) \leq Q, \\
& p \leq \mathscr{V}^{(n)}(\lambda) \leq P, \quad \forall \lambda \in[0, \infty) .
\end{aligned}
$$

Then, we have

$$
\begin{aligned}
& \text { (i) } p \frac{\vartheta^{n-\delta}(\lambda)}{\Gamma(n-\delta+1)}\left(\mathscr{D}_{0^{+}}^{9, \rho} \mathscr{U}(\lambda)\right)+Q \frac{\vartheta^{n-\varrho}(\lambda)}{\Gamma(n-\varrho+1)}\left(\mathscr{D}_{0^{+}}^{9, \delta} \mathscr{V}(\lambda)\right) \\
& \geq p Q \frac{9^{n-\delta}(\lambda)}{\Gamma(n-\delta+1)} \frac{9^{n-\varrho}(\lambda)}{\Gamma(n-\varrho+1)}+{ }^{c} \mathscr{D}_{0^{+}}^{9, \varrho} \mathscr{U}(\lambda)^{c} \mathscr{D}_{0^{+}}^{9, \delta} \mathscr{V}(\lambda), \\
& \text { (ii) } q \frac{\vartheta^{n-\delta}(\lambda)}{\Gamma(n-\delta+1)}\left({ }^{c} \mathscr{D}_{0^{+}}^{9, \varrho} \mathscr{V}(\lambda)\right)+P \frac{9^{n-\varrho}(\lambda)}{\Gamma(n-\varrho+1)}\left({ }^{c} \mathscr{D}_{0^{+}}^{9, \delta} \mathscr{U}(\lambda)\right) \\
& \geq q P \frac{9^{n-\delta}(\lambda)}{\Gamma(n-\delta+1)} \frac{9^{n-\varrho}(\lambda)}{\Gamma(n-\varrho+1)}+\left({ }^{c} \mathscr{D}_{0^{+}}^{9, \delta} \mathscr{U}(\lambda)\right)\left({ }^{c} \mathscr{D}_{0^{+}}^{9, \rho} \mathscr{V}(\lambda)\right), \\
& \text { (iii) } P Q \frac{9^{n-\delta}(\lambda)}{\Gamma(n-\delta+1)} \frac{9^{n-\varrho}(\lambda)}{\Gamma(n-\varrho+1)}+\left({ }^{c} \mathscr{D}_{0^{+}}^{9, \varrho} \mathscr{U}(\lambda)\right)\left({ }^{c} \mathscr{D}_{0^{+}}^{9, \delta} \mathscr{V}(\lambda)\right) \\
& \geq Q \frac{9^{n-\varrho}(\lambda)}{\Gamma(n-\varrho+1)}\left({ }^{c} \mathscr{D}_{0^{+}}^{9, \delta} \mathscr{V}(\lambda)\right)+P \frac{\vartheta^{n-\delta}(\lambda)}{\Gamma(n-\delta+1)}\left({ }^{c} \mathscr{D}_{0^{+}}^{9, \varrho} \mathscr{U}(\lambda)\right), \\
& \text { (iv) } p q \frac{9^{n-\delta}(\lambda)}{\Gamma(n-\delta+1)} \frac{9^{n-\varrho}(\lambda)}{\Gamma(n-\varrho+1)}+\left({ }^{c} \mathscr{D}_{0^{+}}^{9, \rho} \mathscr{U}(\lambda)\right)\left({ }^{c} \mathscr{D}_{0^{+}}^{9, \delta} \mathscr{V}(\lambda)\right) \\
& \geq q \frac{9^{n-\varrho}(\lambda)}{\Gamma(n-\varrho+1)}\left({ }^{c} \mathscr{D}_{0^{+}}^{9, \delta} \mathscr{V}(\lambda)\right)+p \frac{9^{n-\delta}(\lambda)}{\Gamma(n-\delta+1)}\left({ }^{c} \mathscr{D}_{0^{+}}^{9, \varrho} \mathcal{U}(\lambda)\right) .
\end{aligned}
$$

Corollary 15. Let $\quad \mathscr{U}, \mathscr{V} \in L_{1}([0, \infty]) \quad$ and $\vartheta(\lambda)=\lambda, \lambda>0, \varrho>0, \delta>0$. Suppose that there exist real constants $q, Q, p, P$, such that

$$
\begin{array}{r}
q \leq \mathscr{U}^{(n)}(\lambda) \leq Q, \\
p \leq \mathscr{V}^{(n)}(\lambda) \leq P, \quad \forall \lambda \in[0, \infty) .
\end{array}
$$

Then, we have new inequalities for Caputo fractional derivative operator:

$$
\begin{aligned}
& \text { (a) }{ }^{c} \mathscr{D}_{0^{+}}^{\delta} \varphi_{1}(\lambda)^{c} \mathscr{D}_{0^{+}}^{\rho} \mathscr{U}(\lambda)+{ }^{c} \mathscr{D}_{0^{+}}^{e} \Upsilon_{2}(\lambda)^{c} \mathscr{D}_{0^{+}}^{\delta} \mathscr{V}(\lambda) \\
& \geq^{c} \mathscr{D}_{0^{+}}^{\delta} \varphi_{1}(\lambda)^{c} \mathscr{D}_{0^{+}}^{\varrho} \Upsilon_{2}(\lambda)+{ }^{c} \mathscr{D}_{0^{+}}^{\varrho} \mathscr{U}(\lambda)^{c} \mathscr{D}_{0^{+}}^{\delta} \mathscr{V}(\lambda) \text {, } \\
& \text { (b) }{ }^{c} \mathscr{D}_{0^{+}}^{\delta} \Upsilon_{1}(\lambda)^{c} \mathscr{D}_{0^{+}}^{e} \mathscr{V}(\zeta)+{ }^{c} \mathscr{D}_{0^{+}}^{e} \varphi_{2}(\lambda)^{c} \mathscr{D}_{0^{+}}^{\delta} \mathscr{U}(\lambda) \\
& \geq^{c} \mathscr{D}_{0^{+}}^{\delta} \Upsilon_{1}(\lambda)^{c} \mathscr{D}_{0^{+}}^{\varrho} \varphi_{2}(\lambda)+{ }^{c} \mathscr{D}_{0^{+}}^{\delta} \mathscr{U}(\lambda)^{c} \mathscr{D}_{0^{+}}^{\rho} \mathscr{V}(\lambda) \text {, } \\
& (\mathrm{c})^{c} \mathscr{D}_{0^{+}}^{\rho} \Upsilon_{2}(\lambda)^{c} \mathscr{D}_{0^{+}}^{\delta} \varphi_{2}(\lambda)+{ }^{c} \mathscr{D}_{0^{+}}^{\rho} \mathscr{U}(\lambda)^{c} \mathscr{D}_{0^{+}}^{\delta} \mathscr{V}(\lambda) \\
& \geq^{c} \mathscr{D}_{0^{+}}^{\varrho} \Upsilon_{2}(\lambda)^{c} \mathscr{D}_{0^{+}}^{\delta} \mathscr{V}(\lambda)+{ }^{c} \mathscr{D}_{0^{+}}^{\delta} \varphi_{2}(\lambda)^{c} \mathscr{D}_{0^{+}}^{\varrho} \mathscr{U}(\lambda) \text {, } \\
& \text { (d) }{ }^{c} \mathscr{D}_{0^{+}}^{\rho} \Upsilon_{1}(\lambda)^{c} \mathscr{D}_{0^{+}}^{\delta} \varphi_{1}(\lambda)+{ }^{c} \mathscr{D}_{0^{+}}^{\rho} \mathscr{U}(\lambda)^{c} \mathscr{D}_{0^{+}}^{\delta}(\lambda) \\
& \geq^{c} \mathscr{D}_{0^{+}}^{\varrho} \Upsilon_{1}(\lambda)^{c} \mathscr{D}_{0^{+}}^{\delta} \mathscr{V}(\lambda)+{ }^{c} \mathscr{D}_{0^{+}}^{\delta} \varphi_{1}(\lambda)^{c} \mathscr{D}_{0^{+}}^{\varrho} \mathscr{U}(\lambda) \text {. }
\end{aligned}
$$

Example 1. For $n \in \mathbb{N}, \varrho, \delta>0$, and let there be an absolutely continuous function $\mathscr{U}$ and $\mathscr{V}$ defined on $[0, \infty)$. Also, assume that $\vartheta$ be differentiable and strictly increasing on $[0, \infty)$ with $\vartheta(0)=0$, and $p, q>0$ satisfying $p+q=1$. Then, for $\lambda>0$, one has

$$
\begin{aligned}
& \left(\mathrm{a}^{*}\right) p^{c} \mathscr{D}_{0^{+}}^{9, \delta} \mathscr{U}(\lambda)^{c} \mathscr{D}_{0^{+}}^{9, \rho} \mathscr{V}(\lambda)+q^{c} \mathscr{D}_{0^{+}}^{9, \rho} \mathscr{U}(\lambda)^{c} \mathscr{D}_{0^{+}}^{9, \delta} \mathscr{V}(\gamma) \\
& \geq^{c} \mathscr{D}_{0^{+}}^{9, \delta}\left(\mathcal{U}^{p}(\lambda) \mathscr{V}^{q}(\lambda)\right)^{c} \mathscr{D}_{0^{+}}^{9, \rho}\left(\mathcal{U}^{q}(\lambda) \mathscr{V}^{p}(\lambda)\right) \text {, } \\
& \left(\mathrm{b}^{*}\right) p^{c} \mathscr{D}_{0^{+}}^{9, \delta} \mathscr{U}^{p-1}(\lambda)^{c} \mathscr{D}_{0^{+}}^{9, \rho}\left(\mathscr{U}(\lambda) \mathscr{V}^{q}(\lambda)\right) \\
& +q^{c} \mathscr{D}_{0^{+}}^{9, \rho} \mathscr{V}^{q-1}(\lambda)^{c} \mathscr{D}_{0^{+}}^{9, \delta}\left(\mathscr{U}^{q}(\lambda) \mathscr{V}(\lambda)\right) \\
& \geq^{c} \mathscr{D}_{0^{+}}^{9, \delta} \mathscr{V}^{q}(\lambda)^{c} \mathscr{D}_{0^{+}}^{9,0} \mathscr{U}^{p}(\lambda), \\
& \left(c^{*}\right) p^{c} \mathscr{D}_{0^{+}}^{9, \delta} \mathcal{U}(\lambda)^{c} \mathscr{D}_{0^{+}}^{9, \rho} \mathscr{V}^{(2 / p)}(\lambda) \\
& +q^{c} \mathscr{D}_{0^{+}}^{9, \delta} \mathscr{V}(\lambda)^{c} \mathscr{D}_{0^{+}}^{9, \mathrm{e}} \mathscr{U}^{(2 / p)}(\lambda) \\
& \geq^{c} \mathscr{D}_{0^{+}}^{9, \delta} \mathscr{U}^{p}(\lambda) \mathscr{V}(\lambda)^{c} \mathscr{D}_{0^{+}}^{9,0} \mathscr{V}^{q}(\lambda) \mathcal{U}^{2}(\lambda), \\
& \left(\mathrm{d}^{*}\right) p^{c} \mathscr{D}_{0^{+}}^{9, \delta} \mathscr{U}^{(2 / p)}(\lambda) \mathscr{V}^{q}(\lambda)^{c} \mathscr{D}_{0^{+}}^{9, \mathrm{e}} \mathscr{V}^{p-1}(\lambda) \\
& +q^{c} \mathscr{D}_{0^{+}}^{9, \delta} \mathscr{V}^{q^{-1}}(\lambda)^{c} \mathscr{D}_{0^{+}}^{9, \mathrm{e}} \mathscr{U}^{(2 / p)}(\lambda) \mathscr{V}^{p}(\lambda) \\
& \geq^{c} \mathscr{D}_{0^{+}}^{9, \delta} \mathscr{U}^{2}(\lambda)^{c} \mathscr{D}_{0^{+}}^{9, \rho} \mathscr{V}^{2}(\lambda) \text {. }
\end{aligned}
$$

Proof. From the well-known weighted AM-GM inequality,

$$
p a+q b \geq a^{p} b^{q}, \quad \forall a, b \geq 0, p, q>0, p+q=1 .
$$

By setting $a=\mathscr{U}^{(n)}(r) \mathscr{V}^{(n)}(s) \quad$ and $b=\mathscr{U}^{(n)}(s) \mathscr{V}^{(n)}(r), s, r>1$, we have 


$$
\begin{aligned}
& p \mathcal{U}^{(n)}(r) \mathscr{V}^{(n)}(s)+q \mathcal{U}^{(n)}(s) \mathscr{V}^{(n)}(r) \\
& \geq\left(\mathcal{U}^{(n)}(r) \mathscr{V}^{(n)}(s)\right)^{p}\left(\mathcal{U}^{(n)}(s) \mathscr{V}^{(n)}(r)\right)^{q} .
\end{aligned}
$$

Multiplying both sides of (108) by $\left(\vartheta^{\prime}(s)(\vartheta(\lambda)-\vartheta(s))^{n-\delta-} \quad 1 \vartheta^{\prime}(r)(\vartheta(\lambda)-\vartheta(r))^{n-\varrho^{-1}}\right) \quad / \Gamma(n-$ $\delta) \Gamma(n-\varrho)$, which is positive because $r, s \in(0, \lambda), \lambda>0$, and integrating the resulting identity from 0 to $\lambda$, we have

$$
\begin{aligned}
& \frac{p}{\Gamma(n-\delta) \Gamma(n-\varrho)} \int_{0}^{\lambda} \int_{0}^{\lambda} \vartheta^{\prime}(s)(\vartheta(\lambda)-\vartheta(s))^{n-\delta-1} \vartheta^{\prime}(r)(\vartheta(\lambda)-\vartheta(r))^{n-\varrho-1} \mathcal{U}^{(n)}(r) \mathscr{V}^{(n)}(s) \mathrm{d} r \mathrm{~d} s \\
& +\frac{q}{\Gamma(n-\delta) \Gamma(n-\varrho)} \int_{0}^{\lambda} \int_{0}^{\lambda} \vartheta^{\prime}(s)(\vartheta(\lambda)-\vartheta(s))^{n-\delta-1} \vartheta^{\prime}(r)(\vartheta(\lambda)-\vartheta(r))^{n-\varrho-1} \mathscr{U}^{(n)}(s) \mathscr{V}^{(n)}(r) \mathrm{d} r \mathrm{~d} s \\
& \geq \frac{1}{\Gamma(n-\delta) \Gamma(n-\varrho)} \int_{0}^{\lambda} \int_{0}^{\lambda} \vartheta^{\prime}(s)(\vartheta(\lambda)-\vartheta(s))^{n-\delta-1} \vartheta^{\prime}(r)(\vartheta(\lambda)-\vartheta(r))^{n-\varrho-1} \times\left(\mathcal{U}^{(n)}(r) \mathscr{V}^{(n)}(s)\right)^{p}\left(\mathcal{U}^{(n)}(s) \mathscr{V}^{(n)}(r)\right)^{q} \mathrm{~d} s \mathrm{~d} r
\end{aligned}
$$

We conclude that

$$
p \mathscr{D}_{0^{+}}^{\vartheta, \delta} \mathcal{U}(\lambda) \mathscr{D}_{0^{+}}^{\vartheta, \varrho} \mathscr{V}(\lambda)+q \mathscr{D}_{0^{+}}^{\vartheta, \varrho} \mathscr{U}(\lambda) \mathscr{D}_{0^{+}}^{\vartheta, \delta} \mathscr{V}(\lambda) \geq \mathscr{D}_{0^{+}}^{9, \delta}\left(\mathcal{U}^{p}(\lambda) \mathscr{V}^{q}(\lambda)\right) \mathscr{D}_{0^{+}}^{\vartheta, \rho}\left(\mathcal{U}^{q}(\lambda) \mathscr{V}^{p}(\lambda)\right)
$$

which implies $\left(a^{*}\right)$. The rest of inequalities can be shown in a similar way by the following choice of parameters in AM GM inequality:

$$
\begin{aligned}
\left(\mathrm{b}^{*}\right) a= & \frac{\mathcal{U}^{(n)}(s)}{\mathcal{U}^{(n)}(r)}, b=\frac{\mathscr{V}^{(n)}(r)}{\mathscr{V}^{(n)}(s)}, \\
& \mathcal{U}^{(n)}(r), \mathscr{V}^{(n)}(s) \neq 0, \\
\left(\mathrm{c}^{*}\right) a= & \mathscr{U}^{(n)}(r)\left(\mathscr{V}^{(n)}\right)^{(2 / q)}(s), \\
b= & \left(\mathcal{U}^{(n)}\right)^{(2 / q)}(s) \mathscr{V}^{(n)}(r), \\
\left(\mathrm{d}^{*}\right) a= & \frac{\left(\mathcal{U}^{(n)}\right)^{(2 / p)}(r)}{\mathscr{V}^{(n)}(s)}, \\
b= & \frac{\left(\mathcal{U}^{(n)}\right)^{(2 / q)}(s)}{\mathscr{V}^{(n)}(r)}, \quad \mathscr{V}^{(n)}(r), \mathscr{V}^{(n)}(r) \neq 0 .
\end{aligned}
$$

\section{Conclusion}

The main objective of this paper will be a motivation source for future studies. We established some new generalizations for Hermite-Hadamard type pertaining $n$ th-order differentiability for convex functions via Caputo fractional operator in the $\vartheta$-Hilfer sense. To this date, this is the novel version of the Grüss- and Čebyšev-type inequalities for two synchronous functions via the Caputo fractional derivative in the $\mathfrak{\vartheta}$-Hilfer sense. These estimates, bounds, and inequalities hold for all fractional operators mentioned in Remark 1. We conclude this paper by emphasizing, again, that our main result here, being of a very general in nature, can be specialized to yield numerous interesting fractional integral inequalities. Furthermore, they are expected to find some applications for establishing the uniqueness of solutions in fractional boundary value problems in the fractional partial differential equations.

\section{Data Availability}

No data used in this study.

\section{Conflicts of Interest}

The authors declare that they have no conflicts of interest.

\section{Authors' Contributions}

All authors contributed equally to the writing of this paper. All authors read and approved the final manuscript.

\section{Acknowledgments}

The author T. Abdeljawad would like to thank Prince Sultan University for funding this work through research group Nonlinear Analysis Methods in Applied Mathematics (NAMAM) group number RG-DES-2017-01-17.

\section{References}

[1] A. A. Kilbas, H. M. Sarivastava, and J. J. Trujillo, "Theory and application of fractional differential equation," North-Holland Mathematics Studies, Elsevier Sciences B.V., Amsterdam, Netherlands, 2006.

[2] S. G. Samko, A. A. Kilbas, and O. I. Marichev, Fractional Integrals and Derivatives, Theory and Applications, Edited and with a foreword by S. M. Nikolski, Translated from the 1987 
Russian original, Revised by the authors, Gordon and Breach Science Publishers, Yverdon, Switzerland, 1993.

[3] S. Kumar, K. S. Nisar, R. Kumar, C. Cattani, and B. Samet, "A new Rabotnov fractional-exponential function-based fractional derivative for diffusion equation under external force," Mathematical Methods in the Applied Sciences, pp. 1-12, 2020.

[4] S. Bhatter, A. Mathur, D. Kumar, K. S. Nisar, and J. Singh, "Fractional modified Kawahara equation with Mittag-Leffler law," Chaos, Solitons \& Fractals, vol. 131, Article ID 109508, 2019.

[5] S. Kumar, R. Kumar, J. Singh, K. S. Nisar, and D. Kumar, "An efficient numerical scheme for fractional model of HIV-1 infection of CD4+ T-cells with the effect of antiviral drug therapy," Alexandria Engineering Journal, vol. 59, no. 4, pp. 2053-2064, 2020.

[6] S.-S. Zhou, S. Rashid, S. Rashid et al., "New HermiteHadamard type inequalities for exponentially convex functions and applications," AIMS Mathematics, vol. 5, no. 6, pp. 6874-6901, 2020.

[7] S.-B. Chen, S. Rashid, M. Aslam Noor, R. Ashraf, and Y.-M. Chu, "A new approach on fractional calculus and probability density function," AIMS Mathematics, vol. 5, no. 6, pp. 7041-7054, 2020.

[8] S. Rashid, D. Baleanu, and Y.-M. Chu, "Some new extensions for fractional integral operator having exponential in the Kernel and their applications in physical systems," Open Physics, vol. 18, no. 1, pp. 478-491, 2020.

[9] T. Abdeljawad, S. Rashid, A. A. EL Deeb, Z. Hammouch, and Y.-M. Chu, "Certain new weighted estimates proposing generalized proportional fractional operator in another sense," Advances in Difference Equations, vol. 2020, 2020.

[10] S. Rashid, H. Kalsoom, Z. Hammouch, R. Ashraf, D. Baleanu, and Y.-M. Chu, "New multi-parametrized estimates having pth-order differentiability in fractional calculus for predominating $\hbar$-convex functions in Hilbert space," Symmetry, vol. 12 , no. 2 , p. $222,2020$.

[11] S. Rashid, Z. Hammouch, D. Baleanu, and Y.-M. Chu, "New generalizations in the sense of the weighted non-singular fractional integral operator," Fractals., 2020.

[12] I. Podlubny, Fractional Differential Equations, Academic Press, London, UK, 1999.

[13] R. Hilfer, Applications of Fractional Calculus in Physics, World Scientific, Singapore, 2000.

[14] S. Rashid, A. Khalid, G. Rahman, K. S. Nisar, and Y.-M. Chu, "On new modifications governed by quantum Hahn's integral operator pertaining to fractional calculus," Journal of Function Spaces, vol. 2020, Article ID 8262860, 12 pages, 2020.

[15] T. Abdeljawad, S. Rashid, H. Khan, and Y.-M. Chu, "On new fractional integral inequalities for p-convexity within intervalvalued functions," Advances in Difference Equations, vol. 2020, 2020.

[16] S.-S. Zhou, S. Rashid, F. Jarad, H. Kalsoom, and Y.-M. Chu, "New estimates considering the generalized proportional Hadamard fractional integral operators," Advances in Difference Equations, vol. 2020, 2020.

[17] S. Rashid, F. Jarad, H. Kalsoom, and Y.-M. Chu, "On PólyaSzeg'o and Čebyšev type inequalities via generalized K-fractional integrals," Advances in Difference Equations, vol. 2020, 2020.

[18] R. Khalil, M. Al Horani, A. Yousef, and M. Sababheh, "A new definition of fractional derivative," Journal of Computational and Applied Mathematics, vol. 264, pp. 65-70, 2014.
[19] T. Abdeljawad, "On conformable fractional calculus," Journal of Computational and Applied Mathematics, vol. 279, pp. 57-66, 2015.

[20] F. Jarad, E. Ugurlu, T. Abdeljawad, and D. Baleanu, "On a new class of fractional operators," Advances in Difference Equations, vol. 2017, 2017.

[21] T. Abdeljawad and D. Baleanu, "Monotonicity results for fractional diference operators with discrete exponential kernels," Advances in Difference Equations, vol. 2017, 2017.

[22] R. Almeida, "A Caputo fractional derivative of a function with respect to another function," Communications in Nonlinear Science and Numerical Simulation, vol. 44, pp. 460-481, 2017.

[23] A. Atangana and D. Baleanu, "New fractional derivatives with nonlocal and non-singular kernel: theory and application to heat transfer model," Thermal Science, vol. 20, no. 2, pp. 763-769, 2016.

[24] J. Losada and J. J. Nieto, "Properties of a new fractional derivative without singular kernel," Progress in Fractional Differentiation and Applications, vol. 1, no. 2, pp. 87-92, 2015.

[25] F. Mainardi, "Fractional calculus and waves in linear viscoelasticity," An Introduction to Mathematical Models, World Scientific Publishing Company, Singapore, 2010.

[26] S. Rashid, M. A. Latif, and Z. Hammouch, "Fractional integral inequalities for strongly $\mathrm{h}$-preinvex functions for a kth order differentiable functions," Symmetry, vol. 11, no. 12, p. 1448, 2019.

[27] S. Rashid, T. Abdeljawad, F. Jarad, and M. A. Noor, "Inequalities by means of generalized proportional fractional integral operators with respect to another function," Mathematics, vol. 7, no. 12, p. 1225, 2020.

[28] S. Chu, T. Abdeljawad, F. Jarad, and M. A. Noor, "Some estimates for generalized Riemann-Liouville fractional integrals of exponentially convex functions and their applications," Mathematics, vol. 7, no. 9, p. 807, 2019.

[29] S. Rashid, M. A. Noor, K. I. Noor, and F. Safdar, "Integral inequalities for generalized preinvex functions," Punjab University Journal of Mathematics, vol. 51, no. 10, pp. 77-91, 2019.

[30] S. Rashid, M. A. Noor, K. I. Noor, F. Safdar, and Y.-M. Chu, "Hermite-hadamard type inequalities for the class of convex functions on time scale," Mathematics, vol. 7, no. 10, p. 956, 2019.

[31] S. Rashid, F. Safdar, A. O. Akdemir, M. A. Noor, and K. I. Noor, "Some new fractional integral inequalities for exponentially m-convex functions via extended generalized Mittag-Leffler function," Journal of Inequalities and Applications, vol. 2019, 2019.

[32] S. Rashid, M. A. Noor, K. I. Noor, and A. O. Akdemir, "Some new generalizations for exponentially s-convex functions and inequalities via fractional operators," Fractal and Fractional, vol. 3, no. 2, p. 24, 2019.

[33] S. Belarbi and Z. Dahmani, "On some new fractional integral inequalities," JIPAM. Journal of Inequalities in Pure and Applied Mathematics, vol. 10, no. 3, p. 5, Article ID 86, 2009.

[34] Z. Dahmani, "New inequalities in fractional integrals," International Journal of Nonlinear Science, vol. 9, pp. 493-497, 2010.

[35] S. S. Dragomir and N. T. Diamond, "Integral inequalities of Gr'uss type via Pólya-Szegö and Shisha-Mond results," East Asian Journal on Applied Mathematics, vol. 19, no. 1, pp. 27-39, 2003.

[36] M. Caputo and M. Fabrizio, "A new definition of fractional derivative without singular kernel," Progress in Fractional Differentiation and Applications, vol. 1, no. 2, pp. 73-85, 2015. 
[37] T. U. Khan and M. A. Khan, "Generalized conformable fractional operators," Journal of Computational and Applied Mathematics, vol. 346, pp. 378-389, 2019.

[38] P. L. Chebyshev, "Sur les expressions approximatives des integrales définies par les autres prises entre les mêmes limites," Proceedings of the Math. Soc. Charkov.vol. 2, pp. 93-98, 1882.

[39] G. Grüss, "Uber das Maximum des absoluten Betrages von $(1 /(b-a)) \int_{a}^{b} f(x) g(x) \mathrm{d} x-\left(1 /(b-a)^{2}\right) \quad \int_{a}^{b} f(x) \mathrm{d} x \int_{a}^{b} g$ $(x) \mathrm{d} x$," Mathematische Zeitschrift, vol. 39, no. 1, pp. 215-226, 1935.

[40] Z. Dahmani, O. Mechouar, and S. Brahami, "Certain inequalities related to the Chebyshev's functional involving a Riemann-Liouville operator," Bulletin of Mathematical Analysis and Applications, vol. 3, no. 4, pp. 38-44, 2011.

[41] S. K. Ntouyas, P. Agarwal, and J. Tariboon, "On Pólya-Szegö and Chebyshev types inequalities involving the RiemannLiouville fractional integral operators," Journal of Mathematical Inequalities, vol. 2, no. 2, pp. 491-504, 2016.

[42] E. Set, A. O. Akdemir, and İ. Mumcu, "Hadamard's inequality and its extensions for conformable fractional integrals of any order $\alpha>0$," Creative Mathematics and Informatics, vol. 27, no. 2, pp. 197-206, 2018.

[43] F. Jarad, T. Abdeljawad, and D. Baleanu, "Caputo-type modification of the Hadamard fractional derivatives," Advances in Difference Equations, vol. 2012, Article ID 142, 2012.

[44] J.-F. Li, S. Rashid, J.-B. Liu, A. O. Akdemir, and F. Safdar, "Inequalities involving conformable approach for exponentially convex functions and their applications," Journal of Function Spaces, vol. 2020, Article ID 6517068, 17 pages, 2020.

[45] S. Rashid, R. Ashraf, M. A. Noor, K. I. Noor, and Y.-M. Chu, "New weighted generalizations for differentiable exponentially convex mapping with application," AIMS Mathematics, vol. 5, no. 4, pp. 3525-3546, 2020.

[46] S. Rashid, Z. Hammouch, H. Kalsoom, R. Ashraf, and Y.-M. Chu, "New investigation on the generalized $K$-fractional integral operators," Frontiers of Physics, vol. 8, 2020.

[47] S. Rashid, M. A. Noor, K. I. Noor, and Y.-M. Chu, "Ostrowski type inequalities in the sense of generalized $K$ fractional integral operator for exponentially convex functions," AIMS Mathematics, vol. 5, no. 3, pp. 2629-2645, 2020.

[48] H.-H. Chu, S. Rashid, Z. Hammouch, and Y.-M. Chu, "New fractional estimates for Hermite-Hadamard-Mercer's type inequalities," Alexandria Engineering Journal, vol. 59, no. 5, pp. 3079-3089, 2020.

[49] J.-M. Shen, S. Rashid, M. A. Noor, R. Ashraf, and Y.-M. Chu, "Certain novel estimates within fractional calculus theory on time scales," AIMS Mathematics, vol. 5, no. 6, pp. 6073-6086, 2020.

[50] L. Xu, Y.-M. Chu, S. Rashid, A. A. El-Deeb, and K. S. Nisar, "On new unified bounds for a family of functions via fractional q-calculus theory," Journal of Function Spaces, vol. 2020, Article ID 4984612, 9 pages, 2020.

[51] T. Abdeljawad, S. Rashid, Z. Hammouch, and Y.-M. Chu, "Some new local fractional inequalities associated with generalized (s, m)-convex functions and applications," Advances in Difference Equations, vol. 2020, Article ID 406, 2020.

[52] E. Kacar, Z. Kacar, and H. Yildirim, "Integral inequalities for Riemann-Liouville fractional integrals of a function with respect to another function," Iranian Journal of Mathematical Sciences and Informatics, vol. 13, pp. 1-13, 2018.

[53] U. N. Katugampola, "A new approach to generalized fractional derivatives," Bulletin of Mathematical Analysis and Applications, vol. 6, pp. 1-15, 2014. 